\title{
La reforma de la Política de Cohesión de la Unión Europea en el Horizonte post-2020. Propuestas en perspectiva e impacto potencial para Euskadi*
}

\author{
The Reform of the Cohesion Policy \\ of the European Union in the Post-2020 Horizon. \\ Proposals in Perspective and Potential Impact for Euskadi \\ Rafael Bonete Perales \\ Universidad de Salamanca \\ rbonete@usal.es \\ Rafael Muñoz de Bustillo Llorente \\ Universidad de Salamanca \\ bustillo@usal.es
}

doi: http://dx.doi.org/10.18543/ced-58-2018pp25-67

\begin{abstract}
Sumario: I. Introducción.-II. Crecimiento económico y convergencia social. Breve visión teórica.-III. Las etapas de la política de cohesión de la Unión Europea: deseos y realidades. 1. Desde los inicios hasta 1988. 2. Desde 1989: hacia un menor número de objetivos. 3. Euskadi como beneficiario de la política de cohesión de la UE.-IV. Una visión global de la evolución de la cohesión territorial en la UE.-V. Los elementos del debate y conclusiones.
\end{abstract}

Resumen: El presente artículo tiene como objetivo realizar un recorrido sobre la utilización que Euskadi ha realizado de la política de cohesión de la Unión Europea desde la incorporación de España a la UE y reflexionar sobre el impacto que los cambios esperados de la misma tendrán a partir de 2021. Con esa finalidad y tras ofrecer un breve relato de la justificación teórica de la existencia de una política de convergencia en el marco de la UE, se procede a realizar un repaso relativamente pormenorizado del devenir de la política de cohesión en Euskadi y de los principales programas de los que se ha beneficiado. El artículo concluye explorando los distintos argumentos a favor y en contra de una mayor concentración de la política de cohesión en las regiones más desfavorecidas, opción que de aplicarse a partir de 2021 limitaría todavía más el acceso de Euskadi, que en la actualidad está entre el $20 \%$ de regiones más ricas de la UE, a los hoy denominados Fondos Estructurales y de Inversión Europeos.

* Recibido el 19 de diciembre de 2017 y aceptado el 15 de enero de 2018. 
Palabras clave: política de cohesión; desarrollo regional; convergencia; Euskadi.

Abstract: This paper explores the past utilization of Euskadi of the cohesion funds of the European Union and reflects about the potential impact of the expected changes in the cohesion policy for the next programing period starting in 2021. With that aim, and after briefly reviewing the theoretical justification of the existence of cohesion policy in the context of the European Union, the paper presents a relatively detailed analysis of the programs that have benefited Euskadi in the past. The paper concludes exploring the different arguments that have been used in the debate about the convenience of further concentrating the cohesion policy in the less developed regions of the EU. If this perspective were to be finally adopted from 2021 on, the access of Euskadi to the European structural and investment funds would be further limited as result of being among the $20 \%$ riches regions of the EU.

Keywords: cohesion policy; regional development; convergence; Euskadi.

\section{Introducción}

Le corresponde a la Comisión Europea adoptar en mayo de 2018 la propuesta del nuevo Marco Financiero Plurianual (MFP), en el que se establecen los límites máximos de gastos a respetar por la Comisión, Consejo y Parlamento Europeo. Una parte fundamental de este nuevo MFP depende del contenido que finalmente se le dé a la nueva política de cohesión de la UE. Esta propuesta será debatida ampliamente y el contenido final del MFP, en forma de Reglamento del Consejo a aprobar antes del 31 de diciembre de 2020, tendrá un gran impacto sobre la política de cohesión europea en los Estados miembros. El debate sobre la política europea de cohesión del futuro ya ha comenzado ${ }^{1}$ y los posibles escenarios en los que finalmente se desarrollará la misma tendrán

1 Buenos ejemplos de este debate son John Bachtler et al, Towards Cohesion Policy 4.0: Structural Transformation and Inclusive Growth, (Bruselas: RSA Europe, 2017), Comisión Europea, Documento de reflexión sobre el futuro de las finanzas de la UE (Bruselas: 2017), Comisión Europea, My Region, My Europe, Our Future, Seventh report on economic, social and territorial cohesion (Bruselas: 2017) y Comité de las Regiones, Dictamen del Comité Europeo de las Regiones sobre «el futuro de la política de cohesión después de 2020-Por una política de cohesión europea fuerte y eficiente después de 2020» (Bruselas: 2017). 
sus efectos sobre la convergencia europea y las posibilidades de crecimiento de Euskadi².

Para comprender el impacto de la nueva política europea de cohesión en Euskadi abordaremos brevemente, en primer lugar, las relaciones entre crecimiento económico y convergencia social. A continuación, nos centraremos en las etapas de la política de cohesión de la UE hasta nuestros días, prestando especial atención a la importancia de la misma en Euskadi. Después, presentaremos la evolución global de la cohesión territorial en la UE con la finalidad de contrastar si el proceso de integración económica ha ido acompañado de una dinámica de convergencia socioeconómica entre los Estados miembros y las regiones. Con este marco de referencia, en la siguiente sección, sintetizaremos los elementos del debate sobre el futuro de la política de cohesión más allá de 2020 y presentaremos el escenario de futuro más probable para Euskadi.

\section{Crecimiento económico y convergencia social. Breve visión teórica}

Como en tantos otros aspectos de la realidad económica, la Teoría Económica no ofrece un único relato de qué deberíamos esperar en materia de convergencia de renta a lo largo del tiempo entre países de distinto nivel de renta (o regiones dentro de un mismo país). Por un lado, la Teoría Neoclásica del Crecimiento considera que la tendencia natural de economías con distinto nivel de desarrollo sería la convergencia en niveles de renta per cápita. Esta lectura optimista del devenir de las diferencias de renta con el paso del tiempo obedece al supuesto de productividad marginal decreciente del capital, según el cual, en sociedades con un alto nivel de capitalización, y por lo tanto una elevada relación capital-trabajo, las sucesivas adiciones de capital realizarán aportaciones positivas a la producción pero inferiores que en aquellos lugares con menor dotación de capital y donde éste es más escaso. A partir de este supuesto, y como la propia Teoría Neoclásica asocia la remuneración del capital con su productividad, será de esperar que la rentabilidad del capital sea mayor en aquellas sociedades con menor stock de capital, de lo que se deduce una mayor inversión en las mismas, en parte como resultado del flujo de inversiones de los países ricos a los menos ricos. Esta mayor inversión se traducirá a su vez en un mayor crecimiento de la dotación de capital por trabajador, de lo que se derivará mayor cre-

${ }^{2}$ Una síntesis reciente sobre la competitividad actual de Euskadi y su magnífica posición en el contexto nacional ante la economía del conocicimiento nos la proporciona Ernest Reig, dir., La competitividad de las regiones españolas ante la economía del conocimiento (Bilbao: Fundación BBVA, 2017). 
cimiento de la productividad y del PIB. Como resultado, con el tiempo se produciría convergencia en PIB per cápita. Junto a esta dinámica, otros dos factores pueden contribuir a la aceleración de la convergencia. Por un lado, los países/regiones menos desarrollados pueden beneficiarse de la adopción de tecnologías ya disponibles, que acelerarán su crecimiento. Por el contrario, las regiones más desarrolladas, al haber incorporado ya dichas tecnologías en su sistema productivo, dependerán de la aparición de nuevos desarrollos tecnológicos para acelerar (tecnológicamente) su crecimiento. Este factor, conocido como catch up o alcance tecnológico contribuirá a que el crecimiento asociado a la mayor inversión se produzca con mayor intensidad en los países más atrasados. Es lo que el historiador de la economía Gerschenkron ${ }^{3}$ denominó las «ventajas del atraso». Por último, se puede argumentar que las diferencias de renta entre regiones o países generarán movimientos migratorios desde las regiones menos ricas a las de mayor renta, que, bajo determinadas condiciones ${ }^{4}$, también contribuirán a la convergencia. Este sería uno de los factores, que, por ejemplo, habría contribuido a la convergencia del PIB per cápita de las regiones españolas en las décadas del desarrollismo español 1950-19705.

Desde esta perspectiva, se puede argumentar que la intensidad de esta convergencia «automática» se vería incrementada en aquellos países miembros de la Unión Europea por varias razones. Por un lado, la integración en un contexto de libre circulación de capitales facilitará el movimiento de inversiones de los países ricos a los países de menor renta, con lo que se acelerará el crecimiento de la productividad y PIB per cápita. Como señala Soci ${ }^{6}$, la creación de la Comunidad Económica Europea (CEE) estimuló la actividad de las empresas multinacionales, y su ampliación parece haber atraído capital extranjero tanto de dentro como de fuera de la CEE. Por otro lado, la pertenencia a un espacio común contribuirá también a una mejor transferencia de tecnología, contribuyendo al catch up. Por último, la liber-

3 Alexander Gerschenkron, «Economic backwardness in historical perspective», en The Progress of Underdeveloped Areas, ed. por Berthold Frank Hoselitz (Chicago: University of Chicago Press, 1952), 3-29.

${ }^{4}$ La condición necesaria para que la emigración contribuya por un mero efecto estadístico (reducción de la población) al crecimiento del PIB per cápita y a la convergencia es que dicha dinámica no afecte al PIB, bien porque los emigrantes eran población desempleada, bien porque su puesto de trabajo sea ocupado por otra persona desocupada. Cuestión distinta es el posible efecto que tales movimientos migratorios tengan a largo plazo como resultado de la pérdida de población y capital humano (fuga de cerebros).

5 Véase, por ejemplo, Manuel Martín, «Crecimiento y convergencia económica regional en España, en el largo plazo», Estudios Regionales 54 (1999): 47-65.

6 Anna Soci, «FDI and the Process of European Integration. A Brief Overview», Journal of Economic Integration, vol. 18(4), (2003): 607-625. 
tad de movimiento de trabajadores facilitaría la convergencia por la tercera vía. Junto a ello, la propia política europea, con su respaldo del objetivo de cohesión económica, social y territorial, que se materializa en la política de desarrollo regional (como se denominó en sus inicios), y en la política de cohesión (como se denomina en la actualidad), reforzaría la convergencia actuando sobre aquellas variables, como infraestructuras, que pudieran constituir cuellos de botella al proceso de convergencia. ${ }^{7}$

Desde una perspectiva diametralmente distinta, vinculada a la Teoría del Crecimiento Endógeno ${ }^{8}$, se argumenta que las empresas se benefician de la concentración de la producción como forma de aprovechar las posibles economías de escala (a mayor escala de producción menores costes medios) y de aglomeración (la concentración de actividad productiva de distintas empresas en un mismo lugar genera ventajas de costes) de forma que la concentración de capital dará a lugar a una mayor y no menor productividad, poniendo en marcha un círculo virtuoso de crecimiento. ${ }^{9}$ Esta concentración también tendrá efectos positivos sobre la innovación y el cambio técnico (verdadero motor del crecimiento a largo plazo). En la medida en que la innovación se beneficie de la experiencia, aquellos lugares que concentren mayor actividad económica concentrarán también mayor actividad de innovación, ${ }^{10}$ con lo que las diferencias espaciales de

${ }^{7}$ De hecho, el resultado de convergencia espacial en renta derivado de la Teoría de la Convergencia Neoclásica solo se da bajo el supuesto de que el único factor que diferencia los países sea nivel de renta per cápita, siendo idénticos en todas las demás variables con efecto sobre el crecimiento económico. Un supuesto difícil de explicar ya que si los países son idénticos en todas las variables con efecto sobre el crecimiento menos su renta per cápita, lo esperable sería que también coincidieran en ésta. Es por ello que se habla de convergencia condicional. Bajo esta perspectiva, la actuación sobre cualesquiera de dichas variables contribuirá a facilitar la convergencia real.

8 Peter Howitt, «Endogenous growth theory», en The New Palgrave Dictionary of Economics, 2. ${ }^{a}$ Ed., edi. por Steven Durlauf y Lawrence Blume, 1732-1735.

9 Krugman, uno de los «padres» de la introducción de las consideraciones espaciales en el análisis económico del comercio, cuenta una anécdota que refleja la reticencia de muchos economistas a la hora de introducir en sus modelos algo, como la existencia de economías de escala y aglomeración, que es parte de la experiencia cotidiana de la gente. En un congreso sobre «nueva teoría del crecimiento» un reputado economista retó en un tono beligerante a que se le indicara evidencia que demostrara la importancia económica de las externalidades positivas (un ejemplo de lo cual son las economías de aglomeración) y rendimientos crecientes. La respuesta de Krugman fue directa: las ciudades. Sobre esta cuestión véase Paul Krugman, «The new economic geography, now middle-aged», presentado en la Association of American Geographers el 16 de abril de 2010, https://www.princeton. edu/ pkrugman/aag.pdf.

10 Ello explica la existencia de lugares como Silicon Valley en Palo Alto (California) o Bangalore en el sureño estado de Karnataka en la India, que concentran buena parte de la industria digital en Estados Unidos e India. 
rentas entre distintas regiones/países tenderán a ampliarse con el paso del tiempo a favor de aquellas regiones/países con más actividad económica en el presente.

Desde esta perspectiva, la integración económica, per se, podría contribuir a una mayor concentración de la actividad económica en la medida en que las empresas radicadas en los polos de mayor actividad expulsaran de un mercado ahora integrado a las empresas situadas en regiones menos desarrolladas y con menor concentración de actividad económica. Esta dinámica se vería además reforzada por los impedimentos derivados de la política comercial de la UE a la puesta en marcha de políticas de apoyo a la industria naciente mediante proteccionismo selectivo a las que a menudo recurrieron los países más avanzados durante su proceso de industrialización ${ }^{11}$. De ahí también las excepciones previstas a este tipo de ayudas en el artículo 107 del TFUE ${ }^{12}$. Esta perspectiva, de ser acertada, justificaría todavía más la existencia de una política de cohesión comunitaria, puesto que la propia dinámica de integración contribuiría al aumento de las desigualdades espaciales, lo que exigiría la puesta en marcha de políticas compensatorias para contrarrestar tal dinámica.

Aunque las dos perspectivas teóricas presentadas más arriba conducen a resultados distintos en términos de la dinámica «esperable» de convergencia entre países, algunos autores ${ }^{13}$ han planteado la posibilidad de que ambas se den de forma simultánea, actuando a distintos niveles espaciales. Según esta hipótesis, la integración económica podría simultáneamente contribuir al proceso de convergencia entre los Estados miembros, al tiempo que se mantiene, o incluso aumenta, la concentración espacial dentro de cada país.

Resumiendo, podemos decir que la dinámica de convergencia anticipada por la Teoría Neoclásica de Crecimiento se ve cuestionada por dos factores. El primero de ellos es que las distintas regiones/países se diferencian tanto en su nivel de renta per cápita, como en los valores del resto

${ }^{11}$ Ha-Joon Chang, Kicking Away the Ladder-Development Strategy in Historical Perspective (Londres: Anthem Press, 2002).

12 Art.107. 3: «Podrán considerarse compatibles con el mercado interior: a) las ayudas destinadas a favorecer el desarrollo económico de regiones en las que el nivel de vida sea anormalmente bajo o en las que exista una grave situación de subempleo, así como el de las regiones contempladas en el artículo 349, habida cuenta de su situación estructural, económica y social; (...) c) las ayudas destinadas a facilitar el desarrollo de determinadas actividades o de determinadas regiones económicas, siempre que no alteren las condiciones de los intercambios en forma contraria al interés común»

13 Kurt Geppert y Andreas Stephan, «Regional disparities in the European Union: Convergence and agglomeration», en Papers in Regional Science, Volume 87(2) (2008): 193-217. 
de las variables con impacto sobre el crecimiento (formación de sus trabajadores, infraestructura, calidad de sus instituciones, localización geográfica, etc.), con lo que nada garantiza que la convergencia por sí misma permita a las regiones/países menos desarrollados alcanzar a los países/ regiones más desarrolladas. En este sentido, el proceso de convergencia no sería automático ${ }^{14}$. El segundo de ellos tiene que ver con la existencia de economías de escala y aglomeración que neutralicen la dinámica de rendimientos decrecientes sobre la que se sustenta la Teoría de la Convergencia Neoclásica. En este caso, la dinámica puesta en marcha por la integración conduciría a mayor y no menor concentración de la actividad económica en lugar de convergencia. En los dos casos, hay espacio para la política regional ya sea como mecanismo de actuación sobre aquellas variables complementarias del crecimiento que faciliten una convergencia plena, ya sea como mecanismo de compensación a las fuerzas de concentración que el propio proceso de integración y la creación de un mercado único potenciarían.

\section{Las etapas de la política de cohesión de la Unión Europea: deseos y realidades}

\section{Desde los inicios hasta 1988}

El establecimiento de una política regional comunitaria no fue una prioridad en los trabajos previos a la redacción del Tratado de Roma ${ }^{15}$. En el mismo no hay ningún Título dedicado a la política regional. Varías son las razones que pueden ayudarnos a comprender la ausencia incial de una verdadera política de desarrollo regional en el Tratado de Roma. En primer lugar, hay que destacar que de los seis países fundadores, sólo Italia tenía disparidades regionales importantes, y que finalmente éstas se tuvieron en cuenta en el Protocolo sobre el Mezzogiorno anejo al Tratado de Roma. Los otros cinco países constituían un bloque

14 Juan Diaz et al., Real convergence in the euro area: a long-term perspective (Fráncfort: ECB Occasional Paper Series No 203 / Diciembre 2017).

15 Aunque en la Conferencia de Messina de 1955 se reconoció la existencia de problemas regionales en los seis países fundadores. En el seno del Comité Spaak se discutió el papel que la Comunidad Económica Europea podía tener en el desarrollo regional. Se llegó a considerar la creación de un fondo regional pero finalmente se optó por el Banco Europeo de Inversiones, al coincidir más con el pensamiento económico mayoritario en ese momento. Sobre esta cuestión veáse Norbert Vanhove y Leo Klaassen, Regional Policy: A European Approach (Amesbury: Ashgate Pub Ltd, 1987), 396. 
económico bastante homogéneo ${ }^{16}$, al menos en las regiones ubicadas en el continente europeo. En segundo lugar, se consideró que el propio funcionamiento de las fuerzas del mercado, con la ayuda del incremento esperado del comercio interregional, solucionaría el problema de los desequilibrios regionales. En tercer lugar, se asumió que la movilidad del trabajo desde las regiones menos desarrolladas a las de mayor renta dentro del mismo país o en otro país de la Comunidad aliviaría el problema de desempleo en las primeras, contribuyendo a la convergencia económica. En cuarto lugar, la propia existencia desde la posguerra de políticas de desarrollo regional de ámbito nacional desaconsejó, al afectar a cuestiones tan sensibles como la organización territorial de los Estados y las relaciones entre la administración y el tejido empresarial, trasladar, al menos en el inicio del proceso de integración, este campo de actuación a Bruselas. En quinto lugar, en el Tratado de la CECA ya estaban previstas ayudas y préstamos vinculados a la reconversión industrial y a la adaptación profesional en este sector, y, por lo tanto, se asumió que los casos más llamativos de desequilibrios regionales ya estaban siendo abordados. En sexto lugar, se consideró que el tratamieno favorable de los incentivos regionales de carácter nacional dirigidos a las zonas menos desarrolladas y el apoyo que iba a significar la PAC para las regiones menos avanzadas de la Comunidad serían suficiente para hacer frente al problema de los desequilibrios regionales. Por último, habría que añadir la importancia que se le otorgó al apoyo destinado a las regiones menos desarrolladas dentro de los objetivos del Banco Europeo de Inversiones (BEI) creado y controlado por los Estados miembros, y el protagonismo del Fondo Social Europeo en el correspondiente ajuste de determinados sectores económicos en declive.

Habría que esperar a la primera ampliación de la Comunidad en 1973 para que con la incorporación de Irlanda, con un PIB per cápita inferior al $60 \%$ de la media comunitaria, el argumento de la cierta homogeneidad económica de los Estados miembros dejara de ser válido. La coincidencia temporal con la primera crisis del petróleo y sus efectos devastadores sobre zonas tradicionalmente industriales, muchas de ellas ubicadas en el Reino Unido, y el saldo financiero desfavorable que comenzaría a sufrir este nuevo Estado miembro debido al poco peso de agricultura en su estructura económica y la importancia presupuestaria

${ }^{16}$ Loukas Tsoukalis, The New European Economy (Oxford: Oxford University Press, 1991), 207-208. Una visión menos complaciente de las disparidades regionales en los países fundadores puede encontrarse en Andrés Rodríguez Pose, Reestructuración socioeconómica y desequilibrios regionales en la Unión Europea (Madrid: Instituto de Estudios Económicos, 1993), 47-53. 
de la PAC $^{17}$, propiciaron, finalmente y después de varios años de discusión, el cambio de actitud del Consejo. Prueba de este cambio fue la puesta en funcionamiento en 1975 del Fondo Europeo de Desarrollo Regional (FEDER) como intrumento financiero clave a la hora corregir los desequilibrios regionales de la Comunidad, junto con un BEI más comprometido con la política de cohesión y dotado de más capital gracias a la primera ampliación.

Los escasos fondos asignados al FEDER ${ }^{18}$, en un presupuesto cuya rúbrica fundamental era la PAC, y el hecho de que en los primeros años fuera en realidad un instrumento financiero subsidario de la política regional de los Estados miembros, controlado por los mismos, impidieron que la Comunidad se dotara de una verdadera política regional hasta 1988, una vez el Acta Única Europea, en vigor desde julio de 1987, había incorporado el Título V sobre «la cohesión económica y social» en el Tratado. La incorporación de Grecia ${ }^{19}$ en 1981, con un PIB per cápita inferior al 65\% de la media comunitaria, y cinco años después, las incorporaciones de España y Portugal, con un PIB per cápita inferior al 77\% y 55\% de la media comunitaria, incrementaron de forma muy significativa las disparidades regionales dentro de la Comunidad y el número de países partidarios de una verdadera política de cohesión comunitaria. A partir de ese momento, y en un contexto en el que la correción de las disparidades de renta regionales empezó a considerarse un prerrequisito para avanzar en

${ }^{17}$ Los recursos que el Reino Unido iba a recibir vía FEDER generaría un saldo presupuestario menos desfavorable en la relaciones financieras de este país con la Comunidad, hecho este importante en el contexto del referéndum, celebrado el 5 de junio de 1975, sobre la permanencia o no del Reino Unido en la Comunidad y que finalmente dio un resultado claramente favorable a la permanencia (67\% de los votos). Comenzaba así una práctica que se ha mantenido hasta nuestros días: utilizar los fondos vinculados a la política de desarrollo regional como un flujo de recursos a tener en cuenta para obtener saldos financieros más favorables. Un análisis de la importancia de «la lógica de la compensación» en la historia de la política de cohesión puede consultarse en Eiko Thielemann, «The Price of Europeanization: Why European Regional Policy Initiatives Are a Mixed Blessing», Regional and Federal Studies, vol.12, No.1, (2002): 43-65.

18 Nació con una dotación de 1300 millones de ecus para tres años, lejos, por tanto, de los 3000 milones de ecus considerados necesarios por la Comisión. Inicialmente la dotación del FEDER no superó el 5\% del Presupuesto General de la Comunidades Europeas.

${ }^{19}$ Confirmándose lo que parece una constante en la evolución histórica de la política de cohesión: siempre que ha habido una ampliación ha tenido efectos importantes sobre el contenido de la política de la misma. En este caso, además, se duplicaba el porcentaje de población que habitaba en las regiones con un PIB per capita inferior al 50\% de la media comunitaria según se afirma en Comisión Europea, Vademecum de la reforma de los fondos estructurales comunitarios, (Bruselas-Luxemburgo:1989). 
el proceso de integración europea ${ }^{20}$, la cohesión económica y social se convirtió en un objetivo de la Comunidad.

A las ampliaciones hay que añadir el debate surgido sobre los efectos potencialmente negativos de la realización del mercado único sobre algunas regiones. Ambas cuestiones acentuaron la necesidad, recogida tanto en el Informe Cecchini como en el estudio de Padoa-Schioppa ${ }^{21}$, de disponer de más recursos en el presupuesto comunitario asignados a la política regional y de desarrollar un enfoque global para que la cohesión económica y social ganara peso dentro de los objetivos de la Comunidad ${ }^{22}$.

Pronto se puso de manifiesto que la estructura de las finanzas comunitarias se debía modificar si se quería dar contenido al nuevo impulso integrador que significaba la realización de lo contemplado en el Acta Única ${ }^{23}$. Cada vez había menos dudas sobre el hecho de que el incremento deseado de la importancia de la política de cohesión, como elemento compensador de los potenciales perdedores en un Mercado Único, no era compatible con su escaso peso dentro del presupuesto comunitario.

La cumbre extraordinaria de Bruselas de febrero de $1988^{24}$, ajustó los futuros presupuestos a los nuevos desafíos al incrementar los gastos comunitarios en casi un 30\%, reducir en términos relativos la importancia de la PAC y aumentar los recursos destinados a la política de cohesión. A partir de ese momento la política regional pasó a ser la segunda rúbrica más importante del presupuesto comunitario, sólo superada por la PAC. Hecho éste que beneficiaría de forma especial a España.

20 Ángel de la Fuente y Xavier Vives, «Regional Policy and Spain. Infrastructure and Education as Instruments of Regional Policy: Evidence from Spain», Economic Policy, Vol 10, N. 20 (1995): 11-51 .

${ }^{21}$ Véase Tommaso Padoa-Schiopa, Efficiency, Stability and Equity: A Strategy for the Evolution of the Economic System of the European Community (Oxford: Oxford University Press, 1987). En este estudio se insistía en que los desequilibrios regionales se podían agravar debido a la liberalización del mercado interior.

${ }^{22}$ David Allen, "Cohesion and Structural Funds: Transfers and Trade-Offs», en Policy Making in the European Union, ed.por Helen Wallace y William Wallace (Oxford: Oxford University Press, 2000), polemiza sobre si la política de cohesión se puso en funcionamiento por un verdadero deseo de reducir las disparidades en renta y bienestar entre las regiones europeas, o se creó más bien para incentivar que los países menos desarrollados de la Comunidad participaran en fases superiores de la integración.

${ }^{23}$ Que consolidó en el Tratado CEE el principio de solidaridad entre los Estados miembros, como nos recuerda Comisión Europea (1989),.

${ }^{24}$ En junio de ese mismo año, en la Cumbre de Hannover, se inició una nueva práctica consistente en pactar para varios años el gasto máximo presupuestario entre la Comisión, el Consejo y el Parlamento Europeo, las denominadas Perspectivas Financieras y más tarde Marco Financiero Plurianual, con el objetivo de evitar grandes discusiones presupuestarias anuales y conseguir con ello pacificar el procedimiento presupuestario. 


\section{Desde 1989: hacia un menor número de objetivos}

El cuadro 1 reproduce, de forma resumida, los objetivos y criterios de elegibilidad de las distintas etapas de la política de cohesión europea. El recorrido de las mismas permite observar dos líneas generales de actuación: mantenimiento de los objetivos centrales a lo largo de todo el periodo y concentración de las actuaciones (se pasa de cinco objetivos en 1989 a dos en 2014). Esa concentración de las actuaciones, sin embargo, se ve afectada por la necesidad política de ofrecer «un poco de política de cohesión» a todos los Estados miembros con la finalidad de hacerla más políticamente aceptable y facilitar el respaldo unánime a la hora de aprobar el Marco Financiero Plurianual.

En primer lugar, el objetivo «fundacional» de la política de cohesión, la reducción de las diferencias de rentas entre las regiones de la UE, aparece durante todo el periodo analizado como el objetivo principal con tan solo ligeras variaciones en el criterio de elegibilidad y denominación. El criterio básico de brecha de renta, según el cual la regiones objetivo 1 eran aquellas con un PIB per cápita inferior al 75\% de la media comunitaria, se mantiene estable, aunque según nos acercamos en el tiempo se le añaden otros criterios que facilitan el acceso a los fondos a regiones que superan ese límite como resultado del efecto estadístico provocado por la ampliación a los países del Este y la subsiguiente caída en la renta media de la UE, que hace que algunas regiones superen el umbral del $75 \%$ del PIB pc de la UE (periodo 2007-2013). Este proceso de flexibilización también se manifiesta en la incorporación de un nuevo criterio vinculado a la renta per cápita del país, y no de la región, según el cual serían subvencionables los Estados cuya renta nacional bruta sea inferior al $90 \%$ de la comunitaria, generalizando el criterio empleado de forma original con los países beneficiarios del Fondo de Cohesión vinculado a la creación de la Unión Monetaria Europea (UME). En la misma línea, el último periodo de la política de cohesión, 2014-20, incorpora toda una serie de criterios de elegibilidad para favorecer a las regiones que hubieran superado el criterio clásico de $75 \%$ del PIB pc de la UE. 


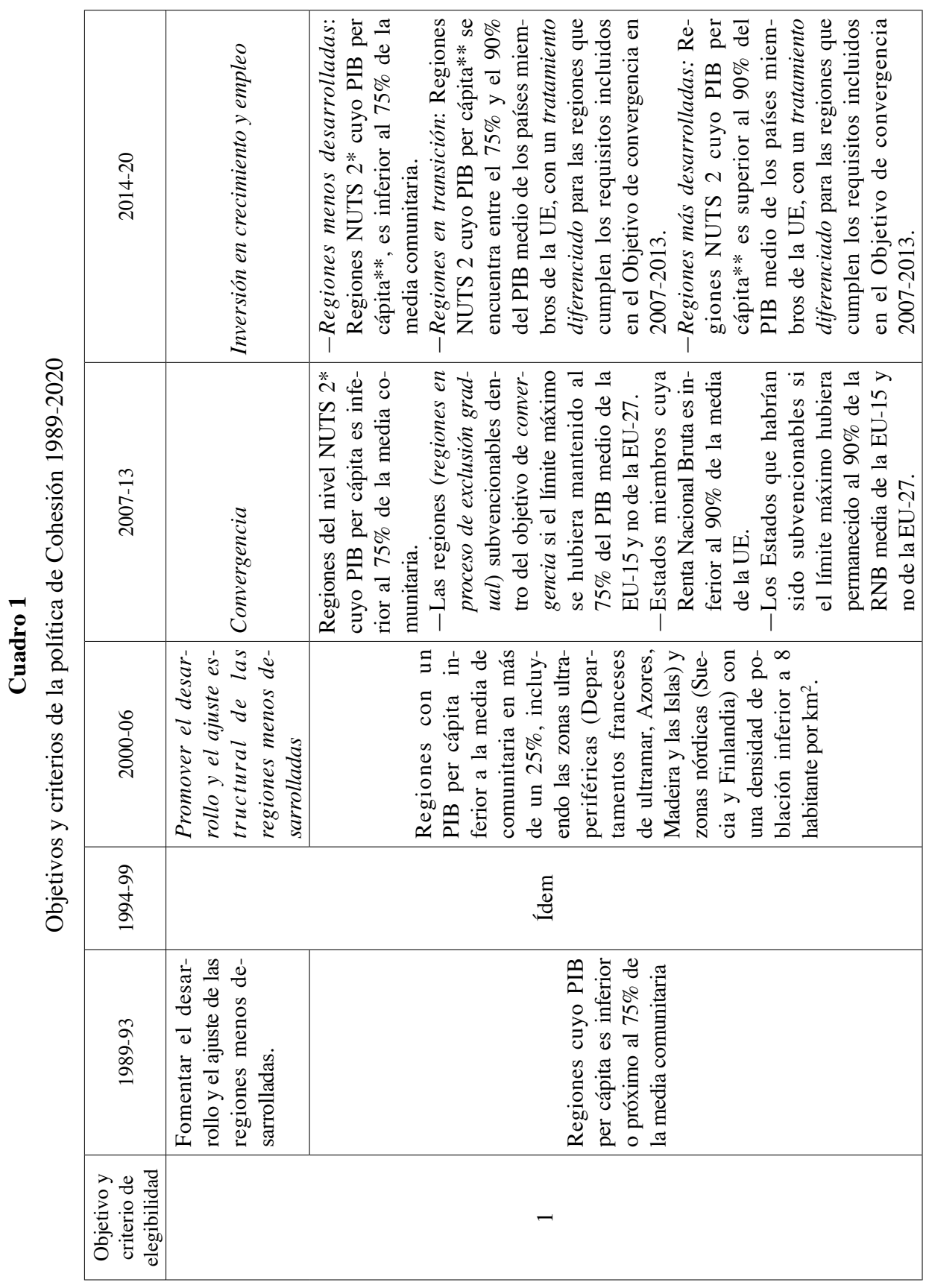




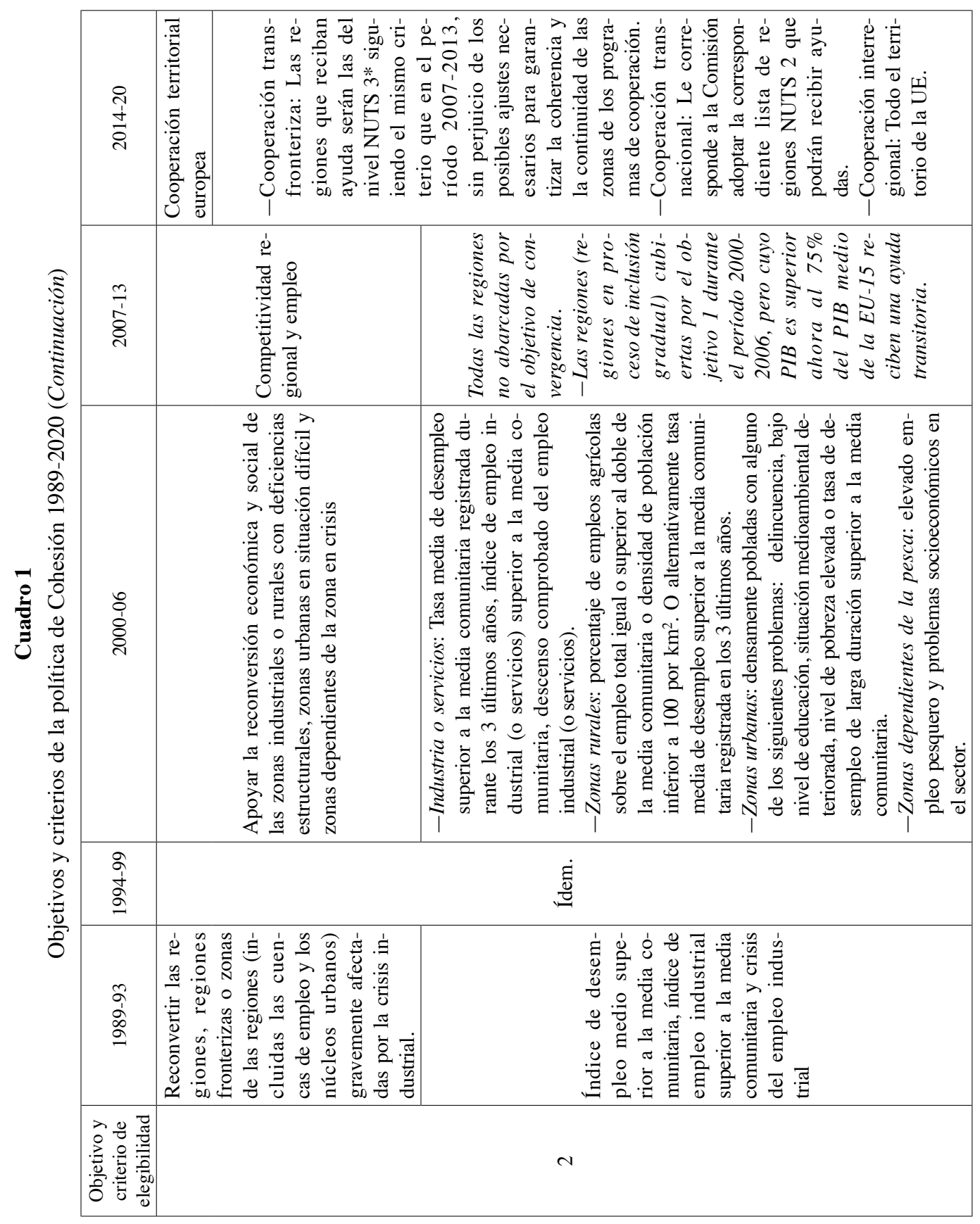




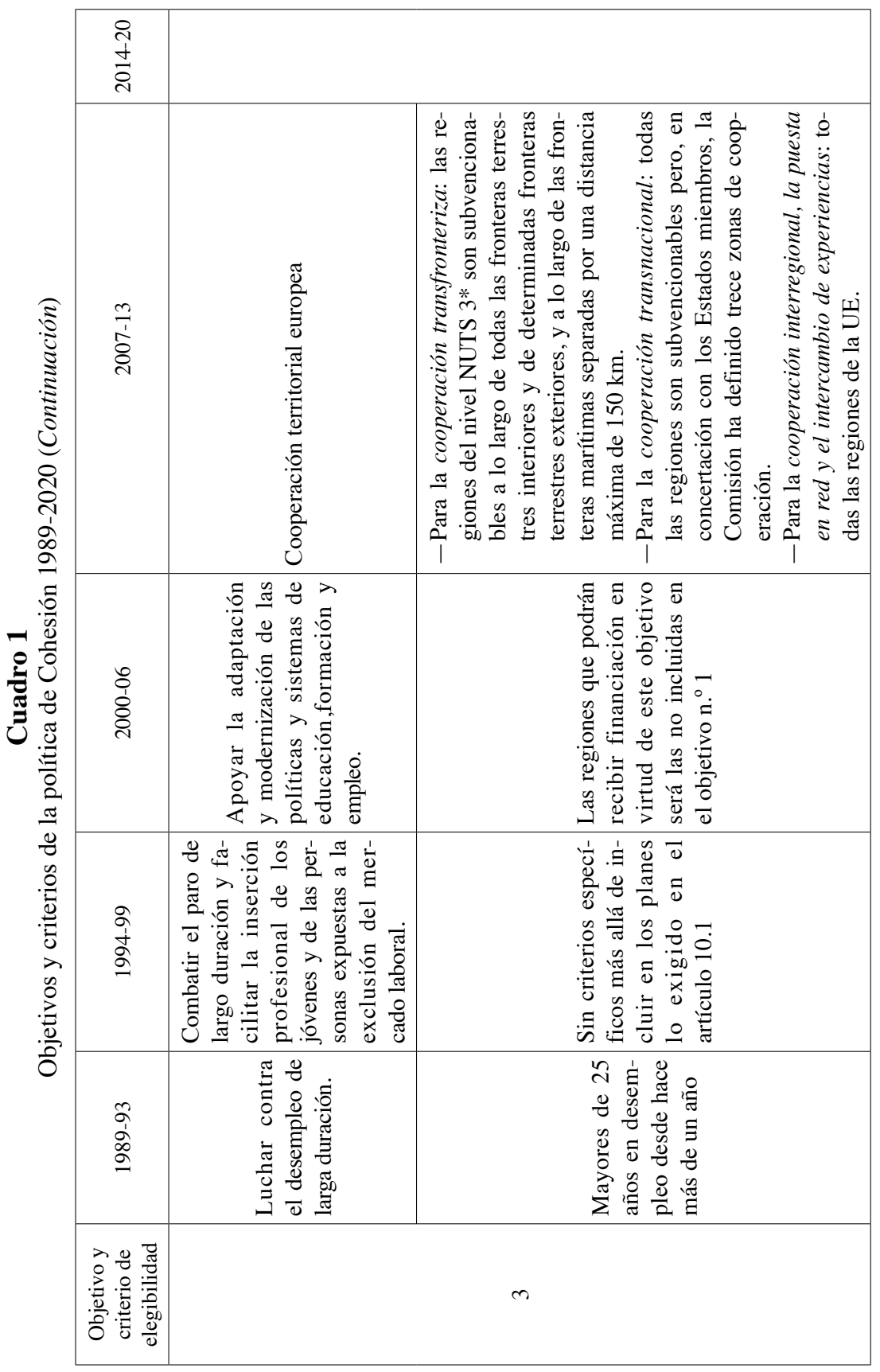




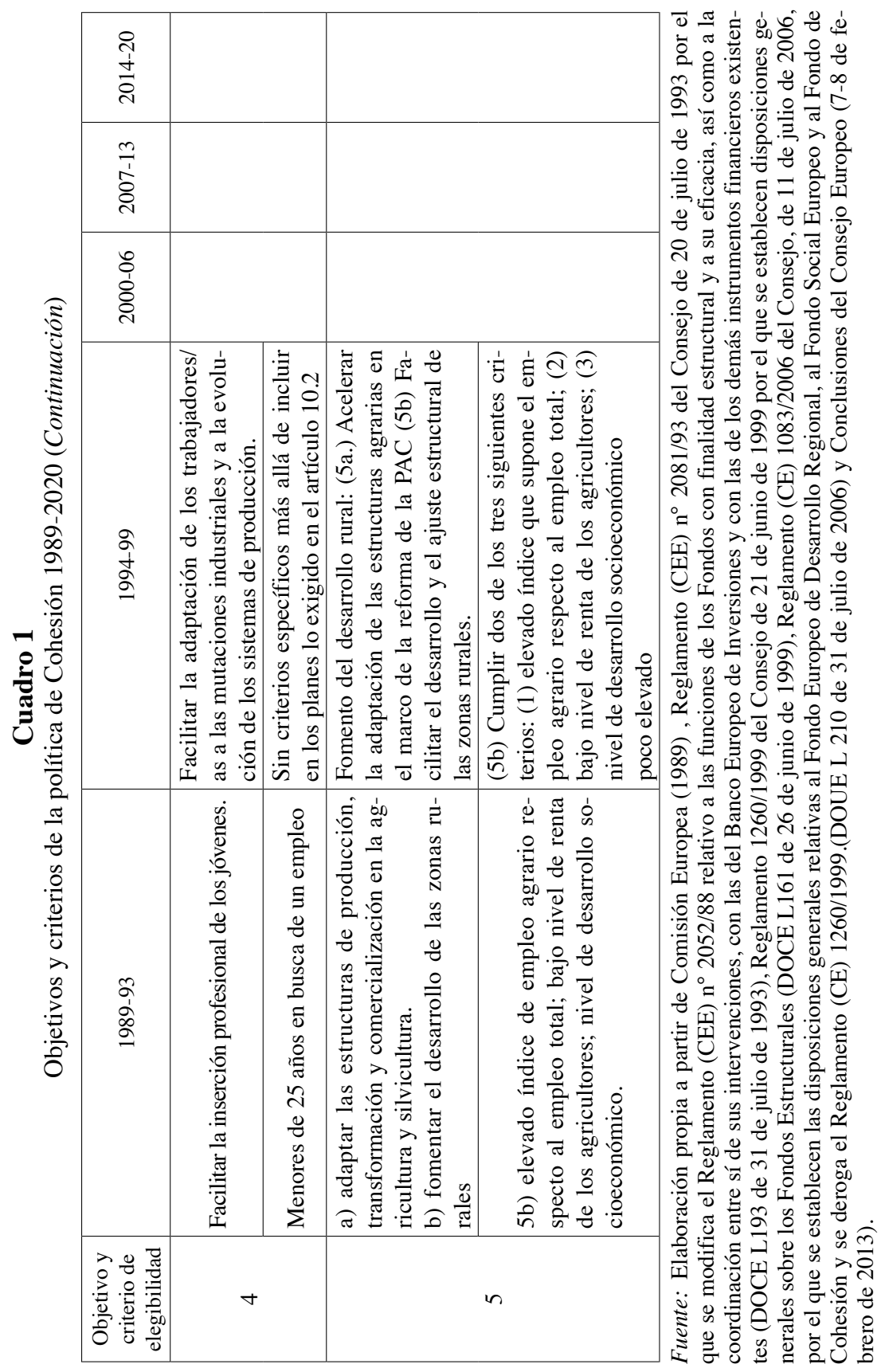


El objetivo dos, fundamental para el caso de Euskadi desde 1986, se plantea desde sus orígenes como un mecanismo de apoyo a zonas (ya sean regiones, regiones fronterizas o zonas de regiones) afectadas gravemente por crisis industriales, independientemente de su localización en una región rica o pobre. Se asume, por lo tanto, que la especificidad espacial de algunas crisis puede hacer que también las regiones de renta alta se vean afectadas por cuestiones de cohesión económica. En este caso, la variable de control es el desempleo y el índice de empleo industrial. Este objetivo se mantiene a lo largo del tiempo si bien desde 2000 se amplía también al sector servicios e incorpora a las zonas rurales (que antes estaban contempladas en el objetivo 5), las dependientes de la pesca y las urbanas con problemas de deterioro socio económico (delincuencia, bajo nivel de educación, situación medioambiental deteriorada, nivel de pobreza elevada o tasa de desempleo de larga duración superior a la media comunitaria). A partir de 2007 cambia de nombre, denominándose competitividad regional y empleo, se simplifica y se amplía al incluir todas las regiones cubiertas por el objetivo 1 durante el período 2000-2006, pero cuyo PIB es superior ahora al $75 \%$ del PIB medio de la EU-15, que pasan a recibir una ayuda transitoria. A partir de 2014 pasa a estar englobado parcialmente dentro de la nueva denominación inversión en crecimiento y empleo. Por último, el objetivo de cooperación territorial europea, creado en 2007, se mantiene a partir de 2014, y sigue centrándose en la cooperación transfronteriza, transnacional e interregional. De este modo en el período actual (2014-2020) la política de cohesión se concentra en tan solo dos objetivos: inversión en crecimiento y empleo y cooperación territorial europea.

El objetivo tres, que también ha propiciado que Euskadi recibiera recursos de la UE, nace con la finalidad de luchar contra el desempleo de larga duración y se mantiene con una formulación similar durante los dos primeros periodos. En 2000, conservando su razón de ser en la lucha contra el desempleo, se transforma para dirigirse a apoyar la adaptación y modernización de las políticas y sistemas de educación, formación y empleo en las regiones excluidas del objetivo 1, pasando a incluirse después estas actuaciones dentro del objetivo competitividad regional y empleo (2007-13) y, más tarde (2014-20) dentro del objetivo inversión en crecimiento y empleo.

El objetivo cuatro del primer periodo, facilitador como el anterior objetivo de la recepción de recursos del presupuesto de la UE también para Euskadi, de carácter horizontal y dirigido a la potenciación de la inserción profesional, se mantienen con algunas variaciones en el periodo 1994-99, pasa a incluirse, en gran parte, en el objetivo 3 durante el período 2000-2006 y termina englobándose, en lo esencial, dentro del objetivo competitividad regional y empleo (2007-13) y, posteriormente (2014-20) dentro del objetivo inversión en crecimiento y empleo. 
Por último, el objetivo cinco, centrado en el mundo rural y en el sector pesquero, a través del cual ha recibido cantidades mucho menores de recursos Euskadi, se mantiene en lo esencial en el período 1994-1999 pero termina siendo absorbido por la política de desarrollo rural (segundo pilar de la PAC) y pesquera (dentro de la política pesquera común) y fuera de la política de cohesión.

\section{Euskadi como beneficiario de la política de cohesión de la UE}

La importancia que la política de cohesión comunitaria ha tenido en Euskadi ha dependido en gran parte del contenido y recursos presupuestarios de la misma a lo largo de los distintos períodos de programación plurianual. De ahí que se haya optado por un relato cronológico sin renunciar a identificar las ideas centrales que han influido sobre la variedad de sectores que durante más 30 años se han beneficiado en Euskadi de la solidaridad europea expresada a través de la política de cohesión.

\section{Periodo 1986-1988}

En el momento de la incorporación de España a la UE, Euskadi estaba inmersa en un proceso intenso de desmantelamiento y reconversión industrial, de ahí que los primeros fondos de desarrollo regional de la Comunidad Económica Europea recibidos en 1988, y canalizados a través del FEDER por un importe equivalente a 16 millones de euros ${ }^{25}$, se centraran sobre todo en ayudas a la reconversión y reestructuración industrial ${ }^{26}$. De hecho, y como reconoce el Gobierno Vasco $^{27}$, la consideración de Euskadi como región industrializada en declive ha sido el principal motivo de la ayuda europea al menos durante el período transcurrido de 1986 a 2011.

25 Aunque en aquel momento no existía el euro y las cantidades eran en ecus, utilizaremos euros también en este período al haberse aplicado en 1999 la equivalencia entre el ecu y el euro.

${ }^{26}$ Aunque el hecho de que entre los proyectos se encontrara la linea I del metro de Bilbao es una prueba de que no sólo se recibieron ayudas destinas a la reconversión y reestructuración industrial.

27 Gobierno Vasco, 25 años de Euskadi en Europa. La ayuda a Euskadi de los fondos estructurales europeos (1986-2011), Departamento de Economía y Hacienda, 2011. Sobre las dificultad de hacer compatibles los intereses de una Comunidad Autónoma como Euskadi en declive industrial y un país de la cohesión como España, véase José Antonio Varela, «Un balance de la política de cohesión europea en Euskadi», Ekonomiaz n. ${ }^{\circ} 82$ (2013): 219-267. 
Además de los recursos destinados al gaseoducto Tolosa-Rentería, al recien creado (1985) Parque Tecnológico de Zamundio ${ }^{28}$ y al impulso del polígono industrial de Abanto-Zierbana, Euskadi se benefició de Iniciativas comunitarias ${ }^{29}$ centradas en la reconversión siderúrgica (RESIDER), naval (RENAVAL), mejora de las telecomunicaciones (STAR) e impulso de la energías renovables (VALOREN). Dentro del programa RESIDER en una primera fase (1988) se canalizaron hacia Euskadi 12 millones de euros, mientras que en el caso del programa RENAVAL habría que esperar a 1992 para que la ayuda del FEDER se concretara en 16,5 millones de euros. Tanto el programa STAR (12 millones de euros) como VALOREN (8 millones) se desarrollaron desde 1987 a 1991 y, por lo tanto, sobre todo en el siguiente período de programación.

\section{Periodo 1989-1993}

Durante el primer periodo de programación plurianual de la nueva política de cohesión ${ }^{30}$ (1989-93), impulsada por la Comisión liderada por Jacques Delors con una visión más estratégica de lo que debía ser una política de cohesión eficaz, en la que se financian programas en lugar de proyectos de carácter anual y en la que hubo un incremento muy significativo ${ }^{31}$ de los recursos a disposición de la política de cohesión, Euskadi recibió a través de los fondos estructurales el equivalente a 511,5 millones de euros. La financiación se centró en los objetivos territoriales 2 y 5b, los objetivos horizontales 3 y 4 y en la ayuda dirigida a las zonas fronterizas a través de la iniciativa INTERREG I y STRIDE (apoyo a la investigación y tecnolo-

${ }^{28}$ Parque tecnológico que también recibiría un apoyo significativo por parte de la UE en el período 1989-93.

${ }^{29}$ La Comisión estaba autorizada para actuar por iniciativa propia a través de Iniciativas comunitarias, las cuales pretende contribuir, además de favorecer la aplicación de determinadas políticas comunitarias, tanto a resolver problemas graves relacionados con la aplicación de otras política comunitarias como a ayudar a resolver problemas comunes a determinadas categorías de regiones.

${ }^{30}$ El contenido detallado de la misma puede consultarse en Comisión de las Comunidades Europeas, (1989). En este nueva política de cohesión, guiada por el principio de concentración, lo que se concreta en que las actuaciones estructurales se concentran en un número determinado de objetivos, hay que destacar también que la preparación, financiación, seguimiento y evaluación de las acciones se rigen por el principio de cooperación.

${ }^{31}$ La cumbre extraordinaria de Bruselas de febrero de 1988, ajustó los futuros presupuestos a los nuevos desafíos al incrementar los gastos comunitarios en casi un $30 \%$, reducir en términos relativos la importancia de la PAC y aumentar los recursos destinados a la política de cohesión, pasando de algo más del $15 \%$ a casi el $31 \%$ del presupuesto comunitario.. Hecho éste que beneficiaría de forma especial a España y a sus comunidades autónomas. 
gía). Algo más del $60 \%$ lo aportó el FEDER, mientras que el FSE aportó el $35,2 \%$ y el FEOGA-Orientación (desarrollo rural), destinado al desarrollo rural, el $1,4 \%$.

A través del FEDER se financiaron actuaciones tan diversas como el apoyo al acceso a los mercados financieros de las PYME de Euskadi, la promoción de la internacionalización de la economía vasca, el incremento de la utilización del gas natural en la industria, comercio y viviendas, el tratamiento de residuos, el impulso de la red de centros tecnológicos del Euskadi, el impulso del Ferrocarril Metropolitano de Bilbao, y la mejora de los aeropuertos de Bilbao y Vitoria-Gasteiz junto con la modernización de los puertos marítimos de Bilbao y Pasajes.

Por su parte, el FSE también financió las actuaciones de su ámbito dentro del objetivo n. $^{\circ} 2$, beneficiando a casi 70000 personas. Los fondos comunitarios fueron destinados sobre todo a la mejora de la formación de empleados y desempleados e impulso en la utilización de los mismos de nuevas tecnologías.

Por último, en este nuevo período de la política de cohesión europea, Euskadi se volvió a beneficiar de las Iniciativas comunitarias que más se adaptaban a su realidad económica y social. Los beneficios derivados de la iniciativa STRIDE (I+D en el ámbito regional) para Euskadi se concretaron en el impulso del Plan Estratégico Tecnológico del País Vasco y en la mejora de los servicios que prestaban los Centros Tecnológicos de esta comunidad autónoma a la industria. Por su parte, INTERREG I (cooperación transfronteriza) se centró durante el período 1991-93 en impulsar la diversificación de la actividad económica en la zona fronteriza ${ }^{32}$ entre Euskadi y Francia y la colaboración bilateral Euskadi-Aquitania ${ }^{33}$.

Euskadi se vio favorecido también por las ayudas recibidas de Europa a través del FSE y centradas en los objetivos 3 y 4 . Entre las múltiples actividades destacaron, además del desarrollo de las Iniciativas comunitarias HORIZON (acceso al mercado laboral de discapacitados y grupos minoritarios), NOW (igualdad de oportunidades para las mujeres en el mercado laboral) y EUROFORM (nuevos tipos de cualificaciones), el apoyo al empleo estable, las acciones para impulsar la empleabilidad de las mujeres, minusválidos y emigrantes.

${ }^{32}$ La Comisión Europea, consciente de los efectos económicos de la desaparición de las aduanas interiores una vez el mercado único fuera una realidad a partir de 1993, impulso un gran número de actuaciones para minimizar los efectos económicos adversos en las regiones fronterizas.

${ }^{33}$ El convenio de colaboración entre el Consejo Regional de Aquitania y Euskadi fue suscrito en Burdeos el 3 de octubre de 1989 por el lehendakari José Antonio Ardanza y por parte francesa por el Presidente del Consejo Regional, Jean Tavernier. 
En el ámbito del mundo rural ${ }^{34}$, el FEOGA-Orientación se centró, sobre todo, en mejorar las infraestructuras, en la creación de empleo y en el aumento de las rentas agrarias. La Iniciativa LEADER (desarrollo rural), desde 1989 y hasta 1990, canalizó, para apoyar a las PYME en el mundo rural, la artesanía y el turismo rural, algo más de un millón de euros.

Dentro del primer período de programación (1989-1993) y si incluimos los tres años previos al mismo, Euskadi recibió de la hoy denominada UE, una media de 102 millones de euros anuales. Si tenemos en cuenta que el Estado español recibió una media de 2.400 millones anualmente entre 1989 y 1993 , y 540 millones entre 1986 y 1988, es evidente que, debido a su elevado nivel de desarrollo, Euskadi no se encontró entre las comunidades autónomas más beneficiadas por la política de cohesión de la UE. Por lo tanto, estamos ante unas cifras modestas $(4,25 \%$ de la totalidad de lo recibido por España entre 1989 y 1993) pero que facilitaron la adaptación de Euskadi a la nueva realidad económica y sentaron las bases para que pudiera aprovecharse de las ventajas derivadas de su participación en el mercado único y recuperar la competitividad de su economía. Adaptación a la que también colaboraría muy pronto el nuevo Fondo de Cohesión ${ }^{35}$ contemplado en el Tratado de la Unión Europea y del que España sería el Estado más beneficiado hasta las ampliaciones de la UE en el siglo XXI.

\section{Periodo 1994-1999}

Durante el siguiente periodo de programación de la política de cohesión $^{36}$ (1994-99), España recibió casi 5.900 millones de euros anuales,

${ }^{34}$ Alrededor del $15 \%$ del territorio de Euskadi y menos del 0,40 de la población.

35 Regulado inicialmente en el Reglamento (CEE) n. ${ }^{\circ}$ 792/93 del Consejo, de 30 de marzo de 1993, por el que se establece un Instrumento Financiero de Cohesión (DOUE, L79 de 1 de abril de 1993).

Este fondo se aprobó para aumentar la probabilidad de que los países menos desarrollados (Grecia, Irlanda, Portugal y España) pudieran acceder a la Unión Económica y Monetaria sin ver frenada su necesaria capacidad inversora en infraestructuras de transporte y medio ambiente y evitar así incurrir en deficits excesivos. Para beneficiarse del Fondo de Cohesión se exige un nivel de renta per cápita inferior al 90\% de la media de la UE, en paridad de poder adquisitivo. En el anexo del propio Reglamento se especifica la siguiente asignación indicativa obtenida teniendo en cuenta toda una serie de criterios (población, PNB per cápita, superficie y otros factores socioeconómicos como las insuficiencias de las infraestructuras de transporte): España: $52 \%$ a 58\% del total; Grecia: $16 \%$ a $20 \%$ del total; Portugal: $16 \%$ a $20 \%$ del total; Irlanda: $7 \%$ a $10 \%$ del total.

${ }^{36}$ Entre las novedades más importantes de este nuevo período y con relevancia para Euskadi, además de la creación del Fondo de Cohesión, de la política de desarrollo regional de la UE pueden destacarse las siguientes: (1) Aumentan de forma muy significativa los recursos presupuestarios asignados a la política de cohesión, hasta acercarse a 177000 millones de ecus (precios de 1999). En el caso de los cuatro países beneficiados también por el Fondo 
mientras que, en Euskadi, aunque durante estos años recibió muchos más fondos que en el período anterior ${ }^{37}$ la media anual no superó los 232 millones de euros, y por lo tanto, en términos relativos $(3,63 \%)$ disminuyó su importancia como receptora de fondos comunitarios dentro del Estado español. En este nuevo período Euskadi se beneficiará de forma significativa del Fondo de Cohesión, alcanzado éste casi el 10\% del total de las ayudas europeas recibidas.

Como en el periodo anterior, y debido a su elevado nivel de desarrollo, Euskadi recibió algo más del 50\% del total por ser una región objetivo n. ${ }^{\circ}$ 2. Mientras que los objetivos 3 y 4 se acercaron al $24 \%$, los objetivos 5 a y 5 b no llegaron el $11.5 \%$ y las Iniciativas comunitarias se acercaron al $6.5 \%$ del total de las ayudas recibidas. La distribución con cargo al FEDER se acercó al 44\% mientras que el FSE aglutinó algo más del 37\%. Como en el anterior periodo, el FEOGA-O tuvo un escaso protagonismo derivado del poco peso económico del mundo rural en Euskadi, canalizando sólo el 4\% del total. Destaca la importancia del apoyo al sector pesquero de Euskadi, el $5 \%$ del total, a través del IFOP.

Gracias a la consideración del $63 \%$ del territorio (aunque engloba el $98 \%$ de la población) como zona objetivo n. ${ }^{\circ} 2$, el FEDER y el FSE pudieron cofinanciar un gran número de actuaciones ${ }^{38}$ dentro de los 5 ejes acordados con la UE: apoyo al empleo y a la competitividad de las empresas, protección y mejora del medio ambiente, apoyo a la investigación y desarrollo, mejora de la red de comunicaciones y el impulso del desarrollo local y urbano.

El FSE además de intervenir en Euskadi por ser en gran parte una región objetivo n. $^{\circ} 2$ también se centró, como en el período anterior, en los objetivos 3 y 4 . El primero de los dos objetivos (lucha contra el desempleo de larga duración), y gracias a una ayuda del FSE de casi 279 millones de euros, terminó beneficiando a algo más de 150.000 personas. El objetivo 4 ,

de Cohesión, los recursos asignados significa una duplicación en términos reales de 1992 a 1999, lo que termina beneficiando a Euskadi. (2) Se crea el Instrumento Financiero de Orientación Pesquera (IFOP) y que va a beneficiar de forma significativa a Euskadi. (3) Se procede a una modificación de los objetivos (véase cuadro 1). En concreto, Se crea un nuevo objetivo 4 encaminado a facilitar la adaptación de los trabajadores a los cambios industriales y que terminará beneficiando a Euskadi. (4) Se amplia el número de Iniciativas comunitarias y esto también terminó beneficiando a Euskadi.

37 Disfrutó de un incremento del $226 \%$.

38 Entre las que destacan la financiación de la ampliación del Puerto Autónomo de Bilbao y de la planta de Mercedes-Benz en Vitoria-Gasteiz, facililtar la ubicación de la nueva Aceria Compacta en los terrenos recuperados de Altos Hornos de Vizcaya, el apoyo a la promoción exterior de los productos vascos, la financiación de actividades de formación profesional y de reciclaje profesional, y la puesta en marcha de 250 proyectos de investigación dirigidos a a cubrir las demandas a medio y largo plazo del sector productivo de Euskadi, 
centrado en la facilitar la inserción profesional de los jóvenes y gestionado desde la Fundación para la Formación Continua (FORCEM), como promotor único en España de este objetivo vinculado al FSE, destinó finalmente casi 49 millones de euros a tal cometido.

En este nuevo período de programación con la incorporación de la Rioja Alavesa, las estribaciones del Gorbea y áreas de Vizcaya (parte de la comarca Arratia-Nervión y parte de la comarca de las Encartaciones) y Guipúzcoa (municipios en el entorno del monte Ernio) se amplió el ámbito territorial de aplicación de los objetivos 5 b en Euskadi y la ayuda comunitaria superó los 20 millones de euros.

En el caso del objetivo 5a, centrado en facilitar la adaptación de las estructuras agrarias en el contexto de la reforma de la PAC, se recibieron casi 36 millones de euros procedentes del FEOGA-Orientación y casi 62 millones de euros, vía el recién creado IFOP, destinados a la mejora del sector pesquero de Euskadi.

Hay que destacar también la relevancia de la ayuda recibida, algo más de 88 millones de euros vía ocho Iniciativas comunitarias, la mitad de ellas (ADAPT, PESCA, URBAN y PYME) nuevas para Euskadi, y la variedad ${ }^{39}$ e importancia de las actuaciones englobadas en las mismas.

Euskadi recibió el 4,85\% de todos de los recursos del Fondo de Cohesión asignados a España ${ }^{40}$, centrándose una parte importante en la financiación de carreteras ${ }^{41} \mathrm{y}$ en proyectos de suministro de agua ${ }^{42}$.

\section{Periodo 2000-2006}

En el siguiente periodo de programación de la política de cohesión de la Unión Europea, en el que se incluyeron importantes novedades ${ }^{43}$, España, por última vez, recibió muchas más ayudas europeas que en los períodos previos, llegando alcanzar 8.900 millones de euros anuales. Por su parte, Euskadi, de-

39 Por ejemplo, en la nueva Iniciativa URBAN se centró en la regeneración urbana y mediambiental de Barakaldo. En el caso de INTERREG II hay que resaltar la ayudas a la construcción del recinto ferial FICOBA en Irún y la continuación del apoyo a las relaciones entre Aquitania y Euskadi. Los fondos vía RENAVAL II financieran,entre otras actuaciones la construcción de Euskalduna en Bilbao.

${ }^{40}$ Superada como beneficaria del Fondo de Cohesión por Cataluña $(18,05 \%)$, Andalucia (15.06), Madrid (8,69\%), Comunidad Valenciana $(8,35)$, Galicia $(7,78)$ y Castilla y León $(7,01)$.

${ }^{41}$ Como la autovía de Guipuzcoa a Navarra y el enlace de Arrizeta.

${ }^{42}$ Como la ayuda de 13 millones de euros para la construcción de la presa de Ibiur con la finalidad de suministrar agua a la comarca de Tolosa.

43 Entre las que destacamos, por su impacto sobre Euskadi, las siguientes:

-Disiminuyen el número de objetivos y se concentran más las intervenciones. Por ejemplo se establece que la población de todas las zonas objetivo 2 no supere el $18 \%$ de la población total de la UE. 
bido entre otras razones a una mayor concentración de la política de cohesión en las regiones menos desarrolladas y la disminución de las Iniciativas comunitarias, no se benefició con la misma intensidad de este incremento superior al 50\% en ayudas recibidas por España, recibiendo una media anual de 205 millones de euros, el 2,3\% del total recibido por España.

Una vez más, el hecho de seguir siendo una región europea objetivo n. ${ }^{\circ} 2$ benefició a Euskadi ${ }^{44}$, aunque menos que en el pasado, y mantuvo al FEDER como el fondo más relevante (alrededor del 43\%), seguido del FSE (31\%), FEOGA-Orientación e IFOP (17\%), Fondo de Cohesión (7,2\%), siendo el resto ayudas vinculadas a las Iniciativas comunitarias.

El FEDER se centró sobre todo en impulsar la modernización de las empresas instaladas en Euskadi ${ }^{45}$, la mejora del medio ambiente ${ }^{46}$, el impulso de la investigación, innovación y desarrollo tecnológico ${ }^{47}$, la mejora del sistema de transporte y comunicaciones ${ }^{48}$ y el desarrollo local y urbano $^{49}$. También intervino el FEDER, aunque aportando sólo 2,6 millones de euros en la financiación del PRAI ${ }^{50}$ País Vasco 2002-2003

Por su parte, el FSE, dentro del objetivo . $^{\circ} 2$, se centró en reforzar el capital humano tanto en investigación como en ciencia y tecnología para lo cual financió la contratación de jóvenes investigadores y doctores, la transferencia de conocimientos de los centros de investigación al tejido empresarial y el apoyo a centros tecnológicos de Euskadi. Dentro del objetivo n. 3 el FSE canalizó casi 255 millones de euros para la inserción e reinserción de desempleados, el refuerzo de la capacidad empresarial, la formación

- Dismiminuye de forma muy significativa el número de Iniciativas comunitarias y la financiación destinada a las mismas, lo que termina perjudicando a Euskadi. Pasan a ser sólo cuatro: INTERREG, URBAN, LEADER y EQUAL.

${ }^{44}$ El hecho de que la aplicación del principio de concentración se concretará en que la población de las regiones objetivo . $^{\circ} 2$ de 1 a UE no podían superar el $18 \%$ de la población de la UE se plasmó en el caso español en que no podía superar el 8\%. Esta restricción tuvo como efecto que una pequeña parte de la población de Euskadi (184.106 habitanes, repartidos entre Vitoria-Gasteiz, Donostia-San Sebastián y Bilbao) se excluyera.

45 Como por ejemplo, Mercedes Benz o ITP (Industria de Turbo Propulsores).

46 Destacando la construcción de estaciones depuradores de aguas residuales y la gestión de residuos sólidos urbanos.

${ }^{47}$ Destinando ayudas para impulsar actividades propias de la Red Vasca de Ciencia y Tecnología y financiar infraestructuras en centros de investigación.

${ }^{48}$ Como la mejora del Puerto de Mutriku o la línea II del Ferrocarril Metropolitano de Bilbao.

49 Destacan entre las múliples actuaciones, la constucción del Museo de Arte Contemporáneo de Vitoria-Gasteiz o la ampliación de la línea de tranvía de Bilbao.

${ }^{50}$ El Programa Regional de Acciones Innovadoras (PRAI) lo propuso la Comisión Europea para apoyar el nacimiento de proyectos innovadores en el ámbito del desarrollo regional. En el caso de Euskadi facilitó el impulso de los Centros de Investigación Cooperativa (CIC) que han hecho en gran parte posible que se configure la BioRegión Vasca y la correspondiente promoción del las biociencias en Euskadi. 
continua de trabajadores, el refuerzo de la educación técnico profesional y del capital humano en investigación, ciencia y tecnología, el incremento de la participación de las mujeres en el mercado de trabajo, la integración laboral de las personas con discapacidad y el fomento de las iniciativas del desarrollo local.

El apoyo de la UE dirigido al mundo rural y el sector pesquero se canalizó una vez más a través del FEOGA-Orientación e IFOP. El primer fondo distribuyó algo más de 120 millones de euros entre una amplia variedad de actuaciones $^{51}$, mientras que el IFOP distribuyó casi 118 millones de euros, sobre todo dirigido a la renovación y modernización de la flota pesquera.

La disminución drástica del número de Iniciativas comunitarias provocó que en Euskadi no se superara los 25 millones asignadas a las mismas, incluyendo INTERREG III, URBAN, LEADER+ y EMPLEO.

Dentro de la intervención del Fondo de Cohesión, con un montante de 105 millones de euros asignados a Euskadi durante este período, se incluyeron 36 proyectos $^{52}$.

\section{Periodo 2007-2013}

La irrupción de la crisis económica en Europa terminará influyendo también en la puesta en práctica de la política de cohesión a lo largo de gran parte del período 2007-2013. Si bien, finalmente, se optó por no introducir cambios radicales en materia de política de cohesión en el período de programación 2007-2013, sí que pueden destacarse algunas novedades por su impacto sobre Euskadi, más allá de los cambios significativos en lo relativo a los objetivos, criterios e instrumentos que pueden intervenir en la política de cohesión ya señalados y recogidos en el cuadro 1 y el hecho de haber acordado una programación más estratégica, con un mayor grado de concentración ${ }^{53}$, más simplificada y descentralizada. En primer lugar, el Fondo de Cohesión, al que durante este período se le van aplicar las mismas normas de programación y gestión que al FEDER y FSE, deja de tener un funcionamiento independiente y pasa a incluirse en el objetivo convergencia. En segundo lugar, se incorpora la cohesión territorial ${ }^{54}$ entre los objetivos funda-

${ }^{51}$ Como, por ejemplo, apoyo a la silvicutura, localización del ganado por GPS y la utilización de las TIC en el sector ovino de leche.

52 Entre los que destacan el muelle 3 del Puerto Autónomo de Bilbao y el acondicionamiento del vertedero de residuos de Bilbao.

53 Prueba de ello es que el objetivo convergencia absorbe algo más del $80 \%$ de los recursos presupuestarios asignados a la política de cohesión y la relevancia presupuestaria de las actuaciones vinculadas a la Estrategia de Lisboa y después a la Estrategia Europa 2020.

54 Aunque habría que esperar a la entrada en vigor del Tratado de Lisboa, el 1 de diciembre del 2009, para verla incluida en el artículo 3.3 de la versión consolidada del Tra- 
mentales de la Unión Europea junto con la cohesión económica y social. En tercer lugar, hay un incremento de situaciones especiales ${ }^{55}$, con objetivos redistributivos, que terminan generando pagos a regiones concretas, países o grupos de países. Como en los anteriores períodos de programación, la exigencia de aprobar por unanimidad las perspectivas financieras, genera este reparto final con el objetivo de obtener el apoyo del los Estados miembros favorecidos. En cuarto lugar, y esto le afectó especialmente a España, hay un número reducido de países que terminan haciéndose cargo en gran medida del coste presupuestario de las ampliaciones de la UE en 2004 y $2007^{56}$, al haberse acordado éstas sin incrementar de forma significativa los recursos prespuestarios destinados a la cohesión. En quinto lugar, se establecen nuevos límites máximos de cofinanciación ${ }^{57}$ y, por último, dentro de esfuerzo de simplificación realizado para este período de programación se opta por no establecer Iniciativas comunitarias ${ }^{58}$ y se excluyen, como también ocurrirá en el siguiente periodo de programación, los fondos dirigidos al desarrollo rural y al sector pesquero de la política de cohesión.

Euskadi, como el resto de España, sufrió una significativa disminución en la recepción de los fondos vinculados a la política de cohesión en este

tado de la UE y los arts. 3, 4, 174 y 175 de la versión consolidada del Tratado de Funcionamiento de la UE.

55 Si en el período 2000-2006 se acordaron hasta 13 supuestos especiales, cuyo importe total alcanzó los 5 millardos de euros, en este período se establecieron 18 supuestos especiales y el importe ha superado los 8,5 millardos de euros. España, con 2.6 millardos e Italia, con 1,4 millardos de euros, son los países que más se han beneficiado de estas ayudas. En el caso de España destaca el fondo específico con el objetivo de financiar el «Programa operativo de $\mathrm{I}+\mathrm{D}+\mathrm{i}$ por y para beneficio de las empresas» por un importe de 2248 millones y destinado a facilitar la convergencia tecnológica de las empresas españolas, sobre todo (75\%) en las regiones menos desarrolladas, recibiendo aquellas regiones que salieron del grupo de los menos desarrolladas (regiones phasing in: Castilla y León, Comunidad Valencian y Canarias) un $15 \%$. El resto de los recursos se destinaron a la Comunidad de Madrid, Cataluña, Aragón, Navarra, la Rioja, Cantabria. Islas Baleares y Euskadi, De ahí que esta última sólo recibiera menos de 30 millones por esa vía (1,3\% del total).

56 En concreto, los primeros perdedores de las nuevas perspectivas financieras para el período 2007-2013 fueron los países de la UE-15, sobre todo España, que dependían en mayor medida de los gastos de la cohesión. Sobre esta cuestión véase Ignacio Zubiri, «Las consecuencias presupuestarias de la ampliación de la Unión Europea», en La ampliación al Este de la Unión Europea, ed. por Ignacio Zubiri (Madrid: Academia Europea de Ciencias y Artes, 2007), 17-94. Finalmente, a los países de la UE-15 se les asignó el 48,6\% de los recursos mientras que a los doce países que se integran en la UE en 2004 y 2007 recibieron el resto.

57 75\% para los objetivos convergencia y cooperación territorial europea. Cuando interviene el Fondo de Cohesión y en las actuaciones en las islas griegas y regiones ultaperiféricas, la cofinanciación alcanza el 85\%. Para el objetivo competitividad regional y empleo, propio de Euskadi, alcanza el $50 \%$.

58 Tanto URBAN II como EQUAL se integran en los objetivos de convergencia y cooperación territorial europea e INTERREG III en el objetivo de cooperación territorial europea. 
nuevo período de programación ${ }^{59}$. Pasaría, además a ser merecedora de los mismos por estar englobada dentro del nuevo objetivo de competividad regional y empleo, destino de sólo el 16\% de los recursos comunitarios destinados a política de cohesión.

Una vez más el FEDER (242,1 millones de euros) y el FSE (154,1 millones de euros) fueron los verdaderos protagonistas de la política de cohesión que, gracias al Fondo Tecnológico creado (29,2 millones de euros), pudo alcanzar los 425,4 millones de euros. Si tenemos en cuenta que la cantidad asignada a España durante este período superó los 35.000 millones de euros ${ }^{60}$, ese evidente que el peso de Euskadi como receptor de la política de cohesión disminuyóde forma notable, reduciéndose al 1,2\% del total recibido por España.

El FEDER en Euskadi se centró en la financiación de múltiples actuaciones ${ }^{61}$ vinculadas a la economía del conocimiento, innovación y desarrollo empresarial. Por su parte el FSE, como en los periodos anteriores, se centró en el fomento del espíritu empresarial, mejora de la adaptabilidad de trabajadores y empresarios, la empleabilidad, inclusión social e igualdad entre hombres y mujeres.

\section{Periodo 2014-2020}

El actual periodo de programación introduce cambios significativos ${ }^{62}$ con relación al previo y tiene a sus disposición menos recursos ${ }^{63}$ (alrededor

59 De un $74 \%$ en el FEDER y FSE.

6026.200 millones de euros (incluidos los 3500 millones de euros provenientes del Fondo de Cohesión) centrados en el objetivo Convergencia, 8500 millones para el objetico competitividad regional y empleo y 559 millones para el objetivo cooperación territorial europea.

${ }^{61}$ Como, por ejemplo, la construcción del Automotive Intelligence Center en Amorebieta-Etxano, y la línea III del Ferrocarril Metropolitano de Bilbao

${ }^{62}$ Entre los que destacan, en primer lugar, una mayor concentración de los recursos destinados sobre todo a las regiones menos desarrolladas, siendo el apoyo prespuestario mucho menor para el caso de las otras dos categorías de regiones: regiones en transición y las más desarrolladas como Euskadi, con una tasa de cofinanciación del 50\%. En segundo lugar, se concentran las prioridades en el cumplimiento de la estrategia Europa 2020 de crecimiento inteligente sostenible e integrador. Opción criticada por Juan Ramón Cuadrado Roura y Tomás Mancha, «Política regional y de cohesión», en Economía de la Unión Europea, coordinado por José María Jordán y Cecilio Tamarit (Madrid: Civitas-Thomson Reuters,2013) 401-429, al poder generar que la búsqueda del crecimiento económico europeo propio de la Estrategia Europa 2020 comprometa la primera prioridad de la política de cohesión que debe seguir siendo la reducción de las desigualdades. En tercer lugar, se incorporan una vez más toda una serie de supuestos especiales generadores de asignaciones especiales y que se concretan en 5800 millones de euros destinados a los países que se han visto particularmente afectados por la crisis económica dentro de la zona euro (Grecia, Portugal, Irlanda, España e Italia). Por último, España deja de recibir recursos vía Fondo de Cohesión.

${ }^{63}$ De los 379243 millones de euros propuestos inicialmente por la Comisión al final se ha acordado asignar a la política de cohesión 325 149, de los cuales 322145 representan los 
de 28.600 millones de euros para el actual período de programación en el caso de España). Ambas realidades han terminado afectando a Euskadi, perteneciente a las regiones más desarrolladas ${ }^{64}$, si bien sus efectos totales seguirán produciéndose más allá de 2020.

En el Acuerdo de Asociación ${ }^{65}$ entre la Comisión Europea y España se defiende una reorientación del gasto en el ámbito de la cohesión ${ }^{66}$ hacia la investigación y la innovación; el apoyo a la PYME; la mejora de la calidad de la enseñanza y la formación; el desarrollo de mercados integradores que fomenten la calidad del empleo y la cohesión social y faciliten el aumento de la productividad; asimismo, se apoya la transición hacia una economía con bajas emisiones de carbono. Esta reorientación del gasto podría haber favorecido incialmente a Euskadi si no se hubiera decidido que las consideradas regiones desarrolladas, en la terminología comunitaria, recibieran sólo algo más de 11.000 millones de euros de las que algo más de $230 \mathrm{mi}-$ llones de euros ${ }^{67}$ irán destinados a Euskadi vía FEDER y FSE ${ }^{68}$.

El Programa Operativo FEDER País Vasco 2014-2020 canalizará fondos europeos por un importe algo superior a 176 millones de euros, de los que 157 se destinarán a potenciar la investigación, el desarrollo tecnológico y la innovación y el resto, sobre todo, a facilitar la transición hacia una economía baja en carbono, el apoyo a las PYME y la mejora del uso y calidad de las TIC. Por su parte, el Programa Operativo del País Vasco FSE 20142020 tiene asignados algo más de 54 millones de euros provenientes del pre-

recursos totales asignados al FEDER, FSE y Fondo de Cohesión y el resto, 3000 millones a la Iniciativa sobre Empleo Juvenil.

${ }^{64}$ Junto con Aragón, Asturias, Baleares, Cantabria, Castilla y León, Cataluña, Ceuta, Comunidad Valenciana, Galicia, La Rioja, Madrid y Navarra.

65 Acuerdo fundamental dado que la ejecución de los ahora denominados Fondos Estructurales y de Inversión Europeos (Fondos EIE, donde se incluyen el FEDER; el FSE, el Fondo de Cohesión, para un número limitado de países entre los que ya no está España; Fondo Europeo de Desarrollo Agrícola, FEADER y el nuevo Fondo Europeo Marítimo y de Pesca, FEMP) se lleva a cabo a través de programas, con arreglo al correspondiente acuerdo de asociación.

66 Véase Comisión Europea, Informe de Posición de los servicios de la Comisión sobre el desarrollo del Acuerdo de Asociación y de programas en España en el período 2014-2020 (Bruselas:2012).

${ }^{67}$ Cifra que se verá algo incrementada gracias al importe que reciba finalmente Euskadi por participar en el Programa Operativo Interreg V A España- Francia-Andorra (POCTEFA) con una cifra total asignada de algo más de 189 millones de euros destinada también a Francia y Andorra además de a Navarra, La Rioja, Huesca, Zaragoza y las cuatro provincias catalanas.

${ }^{68}$ Dejamos fuera el resto de los Fondos Estructurales de Inversión Europeos (Fondos EIE) por no beneficiar ya a España (Fondo de Cohesión) o por formar parte de la política de desarrollo rural de la PAC (FEADER) o de la política de pesca (FEMP) y no formar parte en este período de programación de la política de cohesión. 
supuesto de la UE. El mismo debe centrarse en aumentar la participación en el mercado laboral y mejorar la calidad del empleo, adaptar la cualificación de la población a las necesidades del mercado de trabajo, fomentar el espíritu empresarial y la competitividad de las PYME de reciente creación y la inclusión activa, la igualdad de oportunidades y la no discriminación.

Una vez concluya este período, Euskadi recibirá alrededor del $1 \%$ de toda la ayuda europea asignada a España canalizada a través de la política de cohesión, alcanzando la cifra mínima desde el primer periodo de programación plurianual (1989-93).

\section{Una visión global de la evolución de la cohesión territorial en la UE}

Una vez repasadas las visiones teóricas sobre la dinámica espacial del crecimiento económico esperable en una economía de mercado (convergencia frente a divergencia) y efectuado un recorrido por las fases, prioridades y recursos de la política de cohesión de la UE asignados a Euskadi, procede realizar una somera presentación de lo que ha ocurrido con las diferencias espaciales en renta dentro de la UE y España, así como con la posición relativa de Euskadi en ambos espacios.

En el gráfico 1 se reproduce uno de los indicadores más frecuentemente utilizados para representar la convergencia de PIB per cápita (PIB pc). Nos referimos al coeficiente de variación ${ }^{69}$ del PIB per cápita de las regiones NUTS $2^{70}$. De forma complementaria, el gráfico reproduce la desviación estándar, que reflejaría la evolución de la dispersión absoluta de PIB pc. Como se puede observar, el periodo 2000-2015 se caracteriza por una ligera reducción de la dispersión relativa de renta per cápita hasta el comienzo de la crisis y por su estabilidad desde entonces. Esta reducción de las diferencias relativas en renta per cápita entre las regiones europeas es compatible, como suele ser habitual, con un aumento de las diferencias en términos absolutos, tal y como lo refleja el crecimiento de la desviación estándar del PIB pc de las regiones. Esta misma dinámica se observa cuando el análisis se realiza en términos de países, en vez de regiones.

${ }^{69}$ El coeficiente de variación es un indicador relativo de dispersión de renta, esto es, mide las diferencias relativas de renta y no las absolutas (el coeficiente de variación no cambiará si el PIB de todas las regiones se multiplica por dos, aumentando su distancia absoluta pero no relativa, esto es, su posición relativa con respecto a la media), y por lo tanto recoge una visión más amable de la dispersión.

${ }^{70}$ Eurostat no publica los datos para la NUTS 2 de Inner London. Para esta región solo se presentan los datos correspondientes a la clasificación NUTS 3: Inner London - West e Inner London - East. Al corresponder el PIB pc de Inner London West con el PIB p.c. más elevado de la UE: $167.500 €$ en 2015, se ha optado por excluir esta región del cálculo. 
En la medida en que la convergencia, de producirse, normalmente exige periodos largos de tiempo, es interesante señalar que cuando este mismo ejercicio se realiza para el periodo que comprende las últimas dos décadas del siglo pasado para la UE-1571 observamos una reducción de la dispersión relativa (coeficiente de variación) del PIB pc durante la década de 1980 (alrededor del 10\%) y estabilidad desde entonces.
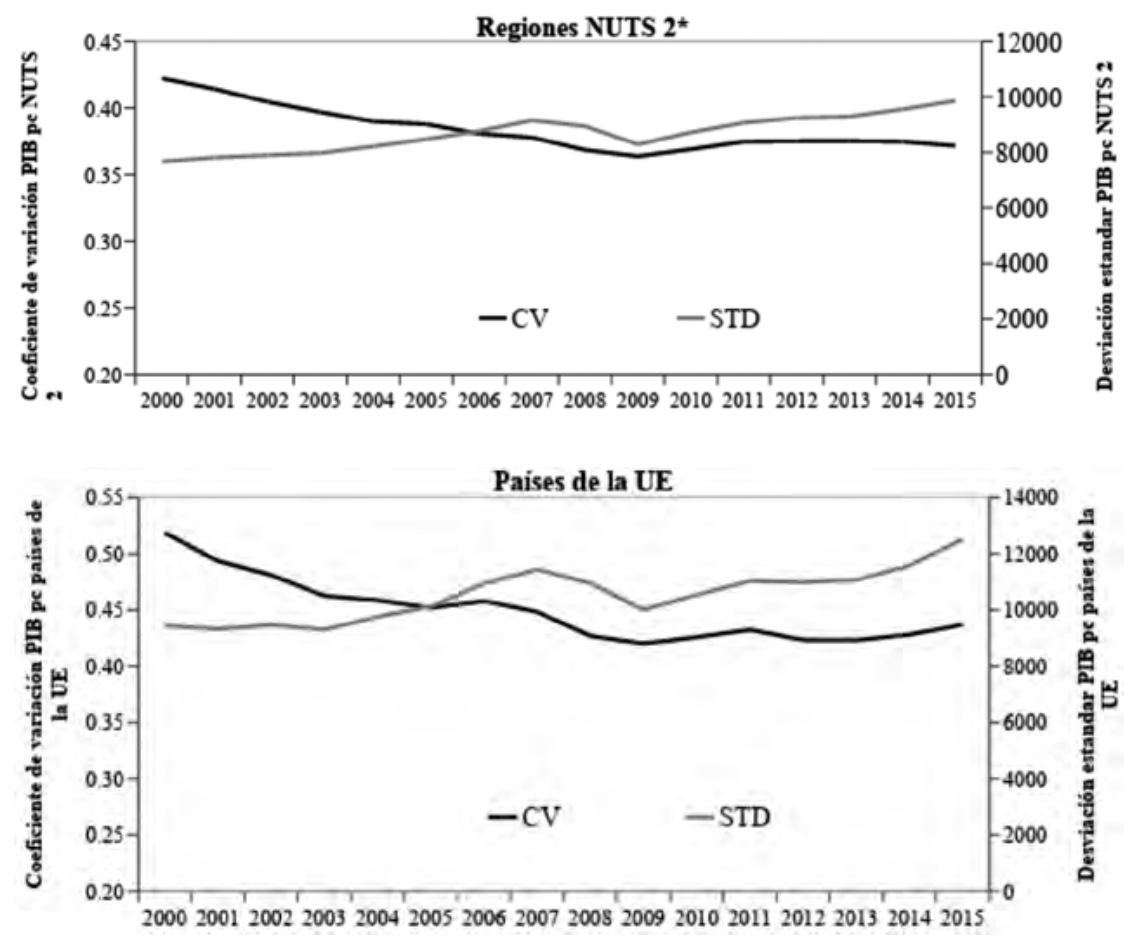

\section{Gráfico 1}

Convergencia especial entre las regiones NUTS 2

y países de la UE (28). 2000-2015.

(*) Excluyendo Inner-London por falta de datos

Fuente: Eurostat y elaboración propia

71 Véase Phillipe Monfort, Convergence of EU regions Measures and evolution, European Union Regional Policy Working Papers, n. ${ }^{\circ} 1$ (2008). 
La realización de este mismo ejercicio para España, en este caso con datos desde 1955 arroja resultados similares en términos de caída de la dispersión relativa de renta per cápita entre las CC.AA. hasta la década de 1980 y estabilidad desde entonces (Gráfico 2). Esta caída de dispersión relativa es compatible con un aumento de la dispersión absoluta que se frenaría con el cambio de siglo. ${ }^{72}$

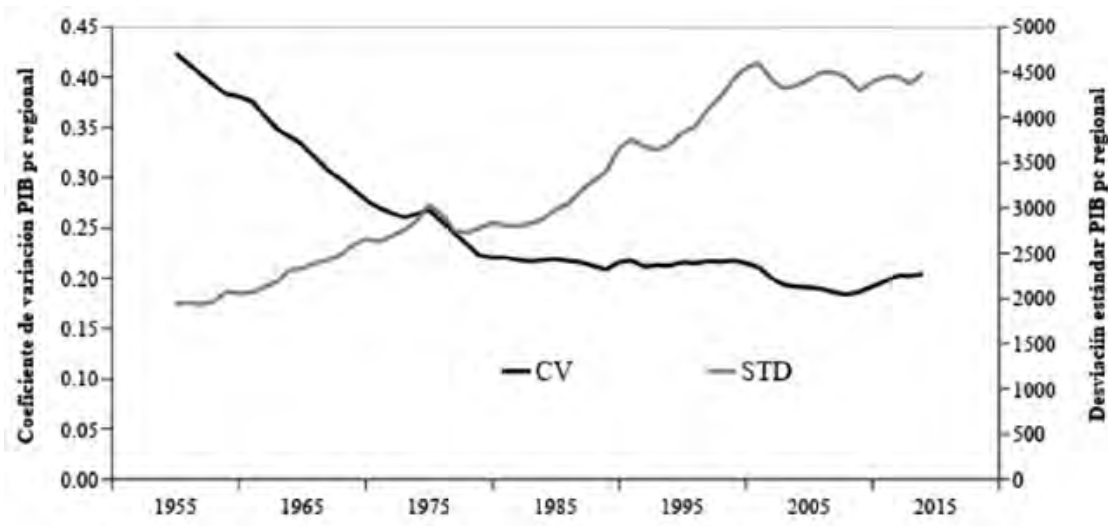

\section{Gráfico 2}

Convergencia regional en España 1955-2014.

Fuente: Elaboración propia a partir de De la Fuente (2017)

Una vez presentada la evolución de la convergencia regional en la UE y España cabe plantearnos en qué posición se sitúa Euskadi y cuál ha sido su evolución en el conjunto de las regiones españolas y europeas. En lo que al primer asunto se refiere, en el Gráfico 3 se observa cómo se ha comportado Euskadi a lo largo de más de medio siglo con respecto a la media nacional (100) y la región con menor y mayor PIB pc de España en cada momento del tiempo: de nuevo encontramos un patrón similar a los referidos más arriba, de reducción de las distancia con respecto a la media hasta la década de 1980, coincidiendo con el periodo de convergencia

72 España nos ofrece un buen ejemplo en lo que se refiere a la perspectiva relativa y absoluta en materia de diferencia de renta: el PIB pc relativo de Andalucía pasa de 70\% de la media en 1957 a 77\% en 2007 (reducción de la dispersión relativa), mientras que la diferencia de PIB pc pasa de algo menos de $1400 €$ a más de $5.500 €$ en idéntico periodo. 
regional, un largo periodo de estabilidad durante el que el PIB pc de Euskadi se sitúa alrededor de un $20 \%$ por encima del de España, y por último el crecimiento de esta diferencia con el cambio de siglo hasta alcanzar el $30 \%$ en 2014, situándose como la segunda CC.AA. en términos de PIB pc por detrás de Madrid.

En términos europeos, Euskadi se situaba en 2015 en el lugar 51 de las 272 regiones NUTS 2 existentes en la UE, lo que le sitúa dentro del 20\% de regiones más ricas en términos de PIB pc de la UE, habiéndose producido una ligera mejoría en su clasificación desde el cambio de siglo (el 2003 ocupaba el lugar 55). Esta realidad, estar entre las regiones más ricas de la UE, sin duda alguna, tendrá efectos significativos a partir de 2021 si finalmente, como es bastante probable, se opta por concentrar más la política de cohesión en las regiones menos desarrolladas de la UE.

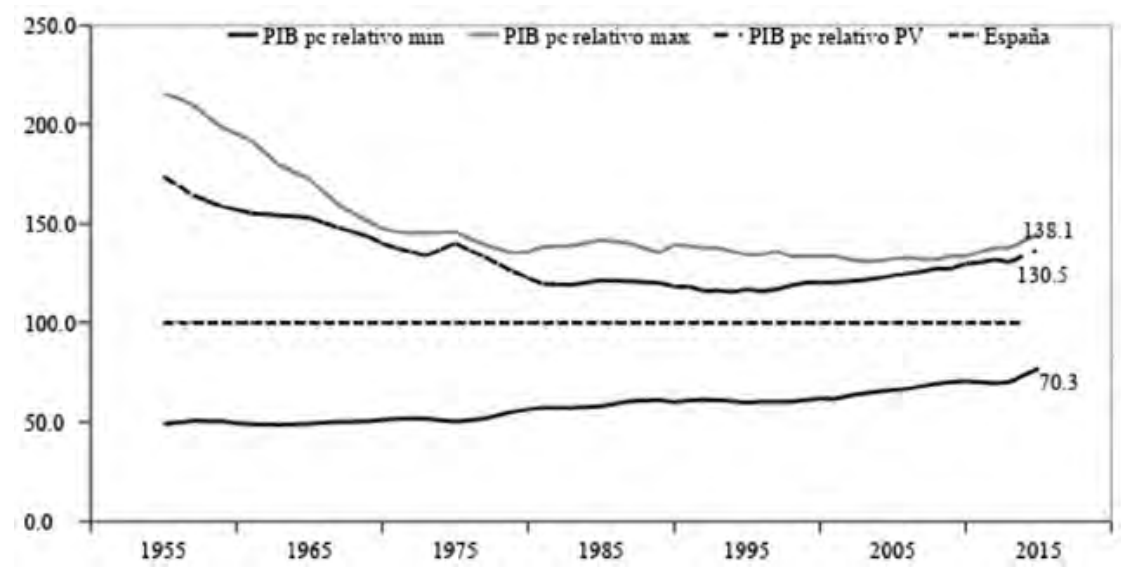

Gráfico 3

Índice de PIB pc Euskadi, región más rica y región más pobre de España (España $=100)$. 1955-2014.

Fuente: Elaboración propia a partir de De la Fuente (2017)

Para acabar esta sección se ha considerado interesante acudir a los estudios que han intentado estimar cuál habría sido el papel de la política de cohesión europea en las dinámicas de convergencia representadas más arriba. En lo que a esto respecta, lo primero que hay que señalar es que la convergencia detectada (o su ausencia, según el periodo observado) no se puede 
achacar directamente a la política de cohesión, ya que la misma responde a múltiples factores que van desde la propia dinámica de crecimiento del país a la política de cohesión nacional o el impacto sobre las distintas regiones del proceso de globalización. Es por ello que para estudiar el efecto de la política de cohesión sea necesario recurrir a la construcción de modelos de simulación que nos permitan comparar la evolución de las regiones europeas con la que hubieran tenido en ausencia de política de cohesión, esto es con un contrafactual construido con ayuda del propio modelo. La Unión Europea realiza desde hace tiempo este tipo de ejercicios mediante el uso de distintos modelos de simulación. Según éstos, para los otrora denominados países de cohesión, Irlanda, Portugal, España y Grecia, como se recoge en el Cuadro 2, se estima que la política de cohesión habría tenido un impacto significativo sobre su crecimiento. De acuerdo con uno de los modelos macroeconómicos utilizados para hacer este tipo de estimaciones, el modelo QUEST del Ecofin, que arroja unos resultados más modestos que otros modelos alternativos como el HERMIN, la política de cohesión habría contribuido a un mayor crecimiento del PIB en estos países, con impactos que irían, en el primero de los periodos analizados, 1994-1999, del 1,2\% en el caso de España al 3\% en el de Irlanda. El impacto se iría reduciendo según nos acercamos a la actualidad como resultado del propio crecimiento de los países y la reducción de los fondos recibidos. Para el periodo vigente, 2014-2020, la Comisión Europea estima un impacto global de la política de cohesión del 0,4\% del PIB para la UE (28), y del 2,6\% para los nuevos países miembros incorporados a la UE desde 2004, UE (13). En países como Polonia, Letonia, Croacia o Estonia el impacto se situaría alrededor del $2 \%^{73}$.

En términos generales, tanto los trabajos encargados por la Comisión Europea, como los estudios académicos realizados para países concretos $^{74}$ muestran un efecto positivo de la política europea de cohesión, pero escaso en términos de intensidad. Varios son los factores que explicarían este resultado. Por un lado, aunque la política de cohesión ha ganado importancia presupuestaria con el paso del tiempo, salvo la disminución significativa en el período 2014-2020, en términos globales los

${ }^{73}$ Según Comisión Europea, Inversión para el empleo y el crecimiento Promoción del desarrollo y la buena gobernanza en las regiones y ciudades de la UE, Sexto Informe sobre la cohesión económica y territorial (Luxemburgo: 2014).

${ }^{74}$ Para el caso español véase, por ejemplo, Simón Sosvilla-Rivero y José Antonio Herce, La política de cohesión europea y la economía española: evaluación y prospectiva (Madrid: Documento de Trabajo del Real Instituto Elcano,142/2004) y Douglas Yuill et al, «Cohesion policy reform process: some implications for Spain and its regions», Presupuesto y Gasto Público n. $^{\circ} 55$ (2009). 
fondos dedicados a ella son modestos cuando se comparan con la magnitud del problema (o cuando se comparan con las transferencias regionales intranacionales). También se ha criticado su excesiva concentración en infraestructuras, sobre todo en la primera época, con mayor efecto sobre la convergencia entre países que entre regiones ${ }^{75}$, así como la dependencia de los resultados de la (escasa) capacidad institucional ${ }^{76}$ de algunos de los receptores de los fondos ${ }^{77}$. En todo caso, y enlazando con la sección dos, hay que tener en cuenta que, en presencia de economías de escala y aglomeración, la política de cohesión tendrá que compensar la desventaja creciente de las regiones menos favorecidas, con lo que estaríamos en un contexto de blanco móvil, donde éste, las regiones ricas, se aleja cada vez más.

\section{Cuadro 2}

El impacto de la política de Cohesión sobre el PIB*

\begin{tabular}{lccc}
\hline & $1994-1999$ & $2000-2006$ & $2007-2013$ \\
\hline España & 3,0 & 1,8 & 0,7 \\
Grecia & 2,2 & 2,4 & 2,2 \\
Irlanda & 3,0 & 0,5 & - \\
Portugal & 2,3 & 2,0 & 1,8 \\
\hline
\end{tabular}

* Crecimiento adicional del PIB al final del periodo comparado con el PIB en ausencia de programas regionales

Fuente: Bachler y Gorzelak (2007) y Monfort et al. (2016).

75 Según Manuel Martín, «Crecimiento y convergencia económica regional en España, en el largo plazo», Estudios Regionales 54 (1998), las infraestructuras contribuirían a la convergencia de las regiones ricas de los países pobres pero no (o al menos no con la misma intensidad) a la convergencia de los países pobres. Dentro de las infraestructuras, serían aquellas vinculadas con las telecomunicaciones las que tendrían un efecto global mayor.

76 Sobre el hecho de que la existencia de instituciones regionales de calidad haya facilitado, en muchos casos, la mayor efectividad del apoyo europeo, veáse Sjef Ederveen, Henri L.F. de Groot y Richard Nahuis, ¿ «Fertile Soil for Structural Funds? A Panel Data Analysis of the Conditional Effectiveness of European Cohesion Policy», Kyklos, vol. 59(1) (2006) y Benedicta Marziotto, The Growth Effects of EU Cohesion Policy: A Meta-Analysis, Bruegel Working Paper 2012/14 (2012).

77 Véase John Bachtler y Grzegorz Gorzelak, «Reforming EU Cohesion Policy. A reappraisal of the performance of the Structural Funds», Policy Studies, vol. 28(4), (2007). 


\section{Los elementos del debate y conclusiones}

Según ha ido aumentando la importancia de la política de cohesión dentro del gasto del presupuesto comunitario, ha ido tomando fuerza la consideración de que hay países que pierden en sus relaciones financieras con la UE y países que ganan. Lo anterior ha intensificado la necesidad de justificar su valor añadido europeo ${ }^{78}$ y el grado de cobertura territorial de la misma. El avance de la defensa de la austeridad presupuestaria en las finanzas públicas de la UE y los posibles efectos presupuestarios del Brexit ${ }^{79}$ han llevado a cuestionar las distintas partidas del gasto comunitario, y en especial aquellas de mayor importancia como la política de cohesión. Asimismo, queda por determinar el impacto final que tendrá en la estructura del gasto del presupuesto de la UE la implicación en la consecución de los Objetivos de Desarrollo Sostenibles dentro de la Agenda $2030^{80}$ y la lucha de la UE contra el cambio climático. Es muy probable, además, que la hoja de ruta presentada en diciembre de 2017 por la Comisión para profundizar en la Unión Económica y Mone$\operatorname{taria}^{81}$ termine afectando, si finalmente se convierte su contenido en normas jurídicas, a la estructura de gasto del presupuesto de la UE y a la importancia de la política de cohesión en el mismo. Por último, la reciente y polémica vinculación del respeto a los valores de la UE como condición previa para ser receptor de fondos europeos ${ }^{82}$ tampoco facilita una configuración clara de los elementos centrales de la política de cohesión más allá de 2020 .

78 Según el Tribunal de Cuentas, Dictamen $n .^{\circ} 7 / 2011$; (2012/C47/01), 3. «Un requisito previo fundamental para obtener un valor añadido europeo es que el gasto procure a la UE y sus ciudadanos beneficios claros y visibles que no podrían lograrse con el gasto realizado a escala nacional, regional o local exclusivamente». Sobre su aplicación a la política de cohesión, véase Mario Monti (dir), Future Financing of the EU, Final report and recommendations of the High Level Group on Own Resources, diciembre de 2016. .

79 Sobre esta cuestión véase Comisión Europea, My Region, My Europe, Our Future, Seventh report on economic, social and territorial cohesion, (Bruselas: 2017). Sobre las posibles soluciones para hacer frente a la disminución de ingresos derivados de la salida del Reino Unido de la UE, véase Gabriel Felbermayr, Clemens Fuest, Jasmin Gröschl y Daniel Stöhlker, Economic Effects of Brexit on the European Economy, Econpol, Policy Report n. ${ }^{\circ} 4$, Noviembre 2017.

${ }^{80}$ Véase Comisión Europea, Documento de reflexión sobre el futuro de las finanzas de la UE (Bruselas: 2017).

81 Véase Comisión Europea, Communication from the Commission to the European Parliament, the European Council, the Council and European Central Bank, Further steps towards completing Europe's Economic and Monetary Unions: a roadmap (Bruselas:2017).

${ }^{82}$ Sobre esta cuestión,véase Jasna Selih, Ian Bond y Carl Dolan, Can the EU funds promote the rule of law in Europe? (Londres: CER, noviembre de 2017). 
Entre los múltiples elementos del debate sobre la futura política de cohesión nos centraremos en el relativo a la oportunidad de la concentrar de la política de cohesión en las regiones menos desarrolladas o en los países menos desarrollados ${ }^{83}$. Dentro de este debate, fundamental en caso de Euskadi dado su elevado nivel de desarrollo, que muy probablemente emergerá otra vez en el proceso de elaboración del próximo Marco Financiero Plurianual a aplicar a partir de 2021 y cuyas ideas básicas tendrá que recoger la Comisión antes de que finalice 2018, destacan entre los argumentos en contra de que los países y regiones desarrolladas de la UE reciban ayuda vía política de cohesión los siguientes ${ }^{84}$ :

- Los países ricos y las regiones ricas de la UE tienen suficiente capacidad financiera e institucional para hacerse cargo de la financiación y seguimiento de su propia política de desarrollo regional ${ }^{85}$.

- Se podría disponer de más recursos para los Estados menos desarrollados de la UE y aumentar así el grado de concentración de la política de cohesión

- Los países desarrollados y las regiones desarrolladas, en su caso, tendrían más libertad para poner en práctica una política regional más acorde a sus intereses sin estar sujetos a las restricciones provenientes de la UE.

- Los recursos vinculados a la realización de la estrategia Europa 2020, pero fuera de la política de cohesión, pueden utilizarse, en todo caso, en las regiones y Estados desarrollados de la UE.

${ }^{83}$ Cuestión que ya se planteó en André Sapir (dir) An Agenda for a Growing Europe, Making the EU Economic System Deliver, Report of an Independent High-Level Study Group established on the initiative of the President of the European Commission (Bruselas: 3 de Julio de 2003), conocido como el Informe Sapir, pero que en los últimos años han defendido de forma insistente gobiernos de países como el Reino Unido, Países Bajos y Suecia. Recientemente, Guntram Wolff, Beyond the Juncker and Schäuble visions of Euro-Area Governance, (Bruselas: Policy Brief n. ${ }^{\circ}$ 6, Bruegel, noviembre 2017),7, ha vinculado este debate a la necesidad de disponer de más recursos para configurar un prespuesto de la UE más acorde con un verdadero eurosistema de política fiscal en la zona euro.

${ }^{84}$ Para una desarrollo más amplio de esta cuestión véase Iain Begg, The Future of Cohesion Policy in Richer Regions, Working Papers n. 3 (Bruselas: European Union, Regional Policy, 2009).

85 En ECORYS, CPB y IFO, A study on EU spending, (Bruselas :DG de Presupuestos de la Comisión Europea), 2008, se plantea de forma amplia los criterios a tener en cuenta para que una política de cohesión pase a ser nacional y se concluye que la política regional aplicada en las zonas ricas de la UE no debería ser responsabilidad de la UE. 
- Las regiones y países desarrollados de la UE se benefician ya, y de forma muy significativa, de la demanda de bienes y servicios que genera la política de cohesión en las regiones y países menos desarrollados de la UE.

- En lugar de recursos presupuestarios, en un escenario poco propicio a su incremento, se pueden utilizar los préstamos del BEI para ayudar también a las regiones y Estados miembros desarrollados de la UE.

Sin embargo, hay también un gran número de argumentos a favor de que las regiones ricas y los Estados prósperos perciban recursos presupuestarios por parte de la UE vía política de cohesión ${ }^{86}$. Entre los mismos, los más relevantes son los siguientes:

- Del Tratado de Lisboa ${ }^{87}$ no se deriva la exclusión, como beneficiarios de la política de cohesión, de las regiones desarrolladas o de los Estados miembros desarrollados.

- Una concepción amplia en términos territoriales de la política de cohesión disfruta de un amplio respaldo tanto por parte del Parlamento y el Comité de las Regiones ${ }^{88}$ como, en menor medida, por parte de los ciudadanos europeos ${ }^{89}$.

- Si se quiere que la política de cohesión tenga relevancia en el desarrollo de la estrategia Europa 2020, no se puede excluir a las regiones y Estados miembros desarrollados.

${ }^{86}$ Más allá de la conveniencia de facilitar la relación directa entra todas las regiones de la UE y la Comisión, el posible beneficio que las regiones menos desarrolladas puedan obtener de las efectos positivos de la política de cohesión sobre las regiones y países más desarrollados de la UE y el hecho de que pueda facilitar la aprobación del Marco Financiero Plurianual por parte de todos los Estados miembros, los ricos y los menos desarrollados, como nos recuerda José Antonio Zamora, «¿Qué futuro para la política de cohesión?», Información Comercial Española, n. ${ }^{\circ} 848$ (2009).

${ }^{87}$ En el art. 174 del Tratado de Funcionamiento de la UE no se excluye a las regiones más desarrolladas como beneficiarias de la política de cohesión. En el mismo se contempla que «A fin de promover un desarrollo armonioso del conjunto de la Unión, ésta desarrollará y proseguirá su acción encaminada a reforzar su cohesión económica, social y territorial. La Unión se propondrá, en particular, reducir las diferencias entre los niveles de desarrollo de las diversas regiones y el retraso de las regiones menos favorecidas».

${ }^{88}$ Postura vuelta a confirmar en Comité de las Regiones, Comité de las Regiones, Dictamen del Comité Europeo de las Regiones sobre «el futuro de la política de cohesión después de 2020-Por una política de cohesión europea fuerte y eficiente después de 2020» (Bruselas: 2017),10, donde se defiende que la política de cohesión debe «fomentar la innovación, la competitividad y el crecimiento sostenible en las regiones más fuertes de Europa».

${ }^{89}$ Según el Eurobarómetro, Citizens' awareness and perceptions of EU regional policy, Flash Eurobarometer 452, (Bruselas: 2017), 29, el 53\% de los ciudadanos son partidarios de que la UE se ocupe de todas las regiones y el $43 \%$ defienden que se centre sólo en las regiones pobres. 
- Su no inclusión debilitaría el dinamismo de las regiones transfronterizas $^{90}$, al estar ubicadas, en muchos casos, parcialmente en regiones y Estados miembros desarrollados.

- La inclusión de las regiones y Estados miembros desarrollados entre los beneficiarios de la política de cohesión puede significar un freno a la proliferación de ayudas estatales dirigidas a las zonas más desarrolladas de la UE y, por lo tanto, evitar los efectos negativos de estas ayudas estatales sobre las regiones menos desarrolladas.

- La elevada capacidad administrativa y calidad institucional de las regiones y Estados miembros desarrollados, en un contexto de programación plurianual ajena a vaivenes políticos, permite obtener elevados niveles de eficiencia a la hora de utilizar los recursos presupuestarios provenientes de la UE.

- La recepción de ayudas vía política de cohesión puede facilitar que los países desarrollados receptores de las mismas mejoren su saldo financiero con la UE, y contribuir a que estos países sigan apoyando la existencia de una política de cohesión, siempre que abarque a todo el territorio de la UE. Todo ello en un contexto en el que «el justo retorno» seguirá estando presente en las próximas negociaciones presupuestarias.

En definitiva, hay argumentos tanto a favor como en contra a la hora de defender una política de cohesión amplia en términos territoriales que termine beneficiando a Euskadi. Es evidente que, si en el próximo período de programación se opta por una mayor concentración de la política de cohesión, Euskadi se verá todavía más perjudicada que en el período actual. El hecho de que no se haya publicado la primera versión del nuevo Marco Financiero Plurianual en el momento de escribir estas líneas nos impide conocer la posición de la Comisión en materia de política de cohesión, si bien el resultado final no se conocerá hasta la aprobación definitiva del mismo por el Consejo Europeo probablemente antes de 2021. En todo caso, es muy probable que aumente la concentración de la política de cohesión y que la misma disponga de menos fondos que en este período de programación. En este escenario muy probable de una futura política de cohesión con menos recursos y más territorialmente concentrada, Euskadi, en primer lugar, debe hacer un esfuerzo para apro-

90 En Comisión Europea, My Region, My Europe, Our Future, Seventh report on economic, social and territorial cohesion (Bruselas: 2017), 12. se afirma que «los programas transfronterizos han transformado las zonas fronterizas, ayudando a eliminar fuentes de conflicto y a crear nuevas oportunidades económicas». 
vechar todavía más ${ }^{91}$ la ventana de oportunidad que significa el Plan de Inversiones para la UE (Plan Juncker) y su correspondiente Fondo Europeo para Inversiones Estratégicas (FEIE). En segundo lugar, también debe aprovecharse al máximo la actividad financiera del BEI, significativamente superior a la del FEIE, en Euskadi ${ }^{92}$ y las operaciones de préstamos de ámbito nacional que favorecen a Euskadi, como el préstamo del BEI por un importe de 1030 millones de euros, que se suman a los 1400 millones concedidos en 2012 y 2013, para financiar las inversiones necesarias para hacer realidad la denominada $\mathrm{Y}$ vasca. Por último, se debe hacer un mayor esfuerzo para beneficiarse de programas comunitarios especialmente relevantes para mejorar la productividad de la economía vasca como Horizonte $2020^{93}$ y su sucesor a partir de $2021^{94}$ y el programa COSME, centrado en las PYME, para mejorar la competitividad de las empresas ${ }^{95}$. Por último, haciendo de la necesidad virtud, hay que señalar que la circunstancia de que en el actual período de programación la política de cohesión de la UE haya sido muy poco significativa para Euskadi facilitará la transición hacia un período con una política de cohesión residual.

${ }^{91}$ Se beneficiarán por un importe de 285 millones de euros las empresas vascas Dominion, Sener, Maier, Iberbancos Luzaro y Bankoa. En la actualidad hay en estudio la asignación de 70 millones de euros para financiar operaciones, una de ellas con la Diputación Foral de Bizkaia.

${ }^{92}$ Sólo en 2016 se firmaron préstamos en Euskadi por un importe de 390 millones de euros, mientras que en la actualidad hay préstamos por un importe de 100 millones de euros en estudio.

93 Sólo Cataluña y la Comunidad de Madrid han superado a Euskadi en España, superada únicamente por Alemania, Reino Unido y Francia en la UE. Si bien, la obtención de 331, 6 millones de euros en las convocatorias de 2014, 2015 y 2016, la participación de 221 entidades de Euskadi en los 530 proyectos de I+D+i justifica el elevado grado de satisfacción del Gobierno Vasco y la Agencia Vasca de Innovación (Innobasque), es evidente que la obtención de un total de 805 millones de euros planteado como objetivo en el Plan Ciencia, Tecnología e Innovación de Euskadi 2020 exige un mayor esfuerzo que el realizado hasta ahora.

${ }^{94}$ Aunque el contenido del conocido como Informe Lamy, recogido en Comisión Europea, $L A B-F A B-A P P$, Investing in the European future we want, Report of the independent High Level Group on maximizing the impact of EU Research \& and Innovation Programme (Bruselas: 2017), en el caso de que influya finalmente de forma significativa en el diseño de la futura política de I+D de la UE, puede generar una mayor divergencia en la UE si se concentra sobre todo en aquellos países líderes. En todo caso, la petición, recogida en el mismo informe, de incrementar las sinergias con los fondos estructurales puede abrir nuevas vías de financiación.

95 Dotado de 2300 millones de euros para el período 2014-2020. 


\section{Referencias}

Allen, David. «Cohesion and Structural Funds: Transfers and Trade-Offs». En Policy Making in the European Union. Editado por Helen Wallace y William Wallace. Oxford: Oxford University Press, 2000.

Bachtler, John, Joaquim Oliveira Martins, Peter Wostner y Piotr Zuber. Towards Cohesion Policy 4.0: Structural Transformation and Inclusive Growth. Bruselas: RSA Europe, 2017.

Bachtler, John., y Grzegorz Gorzelak. «Reforming EU Cohesion Policy. A reappraisal of the performance of the Structural Funds». Policy Studies, vol. 28(4), (2007): 309-326.

Begg, Iain. The Future of Cohesion Policy in Richer Regions. Working Papers n. ${ }^{\circ}$ 3, European Union, Regional Policy, 2009.

Comisión de las Comunidades Europeas Vademecum de la reforma de los fondos estructurales comunitarios. Bruselas-Luxemburgo:1989.

Comisión Europea. Informe de Posición de los servicios de la Comisión sobre el desarrollo del Acuerdo de Asociación y de programas en España en el período 2014-2020. Bruselas:2012.

Comisión Europea. Inversión para el empleo y el crecimiento Promoción del desarrollo y la buena gobernanza en las regiones y ciudades de la UE. Sexto Informe sobre la cohesión económica y territorial. Luxemburgo:2014.

Comisión Europea. Documento de reflexión sobre el futuro de las finanzas de la UE. Bruselas: 2017.

Comisión Europea. My Region, My Europe, Our Future, Seventh report on economic, social and territorial cohesion. Bruselas: 2017.

Comisión Europea. $L A B-F A B-A P P$, Investing in the European future we want. Report of the independent High-Level Group on maximizing the impact of EU Research \& and Innovation Programme., Bruselas: 2017.

Comisión Europea. Communication from the Commission to the European Parliament, the European Council, the Council and European Central Bank, Further steps towards completing Europe's Economic and Monetary Unions: a roadmap. Bruselas:2017.

Comité de las Regiones. Dictamen del Comité Europeo de las Regiones sobre «el futuro de la política de cohesión después de 2020-Por una política de cohesión europea fuerte y eficiente después de 2020». Bruselas: 2017.

Cuadrado Roura, Juan Ramón, y Tomás Mancha. «Política regional y de cohesión» en Economía de la Unión Europea, coordinado por José María Jordán y Cecilio Tamarit .Madrid: Civitas-Thomson Reuters, 2013.

De La Fuente, Ángel. y Xavier Vives. «Regional Policy and Spain. Infrastructure and Education as Instruments of Regional Policy: Evidence from Spain». Economic Policy, Vol 10, N. 20 (1995):11-51.

Diaz, Juan Luis, Ettore Dorrucci , Frigyes Ferdinand Heinzy Sona Muzikarova. Real convergence in the euro area: a long-term perspective. Fráncfort: ECB Occasional Paper Series No 203/Diciembre 2017.

Chang, Ha-Joon. Kicking Away the Ladder-Development Strategy in Historical Perspective. Londres: Anthem Press, 2002. 
ECORYS, CPB y IFO. A study on EU spending. Bruselas: DG de Presupuestos de la Comisión Europea, 2008.

Sjef, Ederveen, Henri L.F. de Groot y Richard Nahuis. «Fertile Soil for Structural Funds? A Panel Data Analysis of the Conditional Effectiveness of European Cohesion Policy». Kyklos, vol. 59(2006): 17-42.

Eurobarómetro. Citizens' awareness and perceptions of EU regional policy. Flash Eurobarometer 452. Bruselas: 2017.

Felbermayr, Gabriel, Clemens Fuest, Jasmin Gröschl y Daniel Stöhlker. Economic Effects of Brexit on the European Economy. Econpol, Policy Report n. ${ }^{\circ}$ 4, noviembre 2017.

Gerschenkron, Alexander. «Economic backwardness in historical perspective». En The Progress of Underdeveloped Areas, editado por Berthold Frank Hoselitz, 3-29. Chicago: University of Chicago Press, 1952.

Geppert Kurt, y Stephan, Andreas. «Regional disparities in the European Union: Convergence and agglomeration», Papers in Regional Science, Volumen 87(2),2008:193-217.

Gobierno Vasco. 25 años de Euskadi en Europa. La ayuda a Euskadi de los fondos estructurales europeos (1986-2011). Departamento de Economía y Hacienda, 2011.

Howitt, Peter. (2008). «Endogenous growth theory».En The New Palgrave Dictionary of Economics, 2. ${ }^{a}$ Ed. Editado por Steven Durlauf y Lawrence Blume, 1732-1735.

Krugman, Paul. Geography and Trade. Cambridge: MIT Press,1999.

Krugman Paul. «The new economic geography, now middle-aged». Presentado en la Association of American Geographers el 16 de abril de 2010. https:/www. princeton.edu/ pkrugman/aag.pdf.

Martín, Manuel. «Crecimiento y convergencia económica regional en España, en el largo plazo». Estudios Regionales 54 (1999): 47-65.

Marzinotto, Benedicta. The Growth Effects of EU Cohesion Policy: A Meta-Analysis. Bruegel Working Paper 2012/14 (2012).

Monfort, Phillipe. Convergence of EU regions Measures and evolution. European Union Regional Policy Working Papers, n. ${ }^{\circ} 1$ (2008).

Monfort, Phillipe, Violeta Piculescu, Alexandra Rillaers, Kai Stryczynski, Janos Vargas. The impact of cohesion policy 2007-2013: model simulations with Quest III Final Report. Work Package 14a Ex post evaluation of Cohesion Policy programmes 2007-2013, focusing on the European Regional Development Fund (ERDF) and the Cohesion Fund (CF). European Commission, DirectorateGeneral for Regional and Urban Policy Brussels (2016).

Monti, Mario (dir.). Future Financing of the EU, Final report and recommendations of the High Level Group on Own Resources, diciembre 2016

Reig, Ernest. Dir. La competitividad de las regiones españolas ante la economía del conocimiento. Bilbao: Fundación BBVA, 2017.

Rodríguez Pose, Andrés. Reestructuración socioeconómica y desequilibrios regionales en la Unión Europea. Madrid: Instituto de Estudios Económicos,1993.

Padoa-Schiopa, Tommaso. Efficiency, Stability and Equity: A Strategy for the Evolution of the Economic System of the European Community. Oxford: Oxford University Press, 1987. 
Sapir, André (dir). An Agenda for a Growing Europe, Making the EU Economic System Deliver. Report of an Independent High-Level Study Group established on the initiative of the President of the European Commission. Bruselas: 3 de Julio de 2003.

Selih, Jasna, Ian Bond y Carl Dolan. Can the EU funds promote the rule of law in Europe? Londres, CER, noviembre de 2017.

Sosvilla-Rivero, Simón y José Antonio Herce. La política de cohesión europea y la economía española: evaluación y prospectiva. Madrid: Documento de Trabajo del Real Instituto Elcano,142/2004.

Soci, Anna. «FDI and the Process of European Integration. A Brief Overview», Journal of Economic Integration. vol. 18(4), (2003): 607-625.

Thielemann, Eiko. «The Price of Europeanization: Why European Regional Policy Initiatives Are a Mixed Blessing». Regional and Federal Studies, vol.12, No.1, (2002): 43-65.

Tribunal de Cuentas. Dictamen $n .^{\circ} 7 / 2011$. (2012/C47/01).

Tsoukalis, Loukas. The New European Economy. Oxford: Oxford University Press,1991.

Vanhove, Norbert y Leo Klaassen. Regional Policy: A European Approach, Amesbury: Ashgate Pub Ltd, 1987.

Varela, José Antonio. «Un balance de la política de cohesión europea en Euskadi». Ekonomiaz, n. ${ }^{\circ} 82$ (2013): 219-267.

Zamora, José Antonio. «¿Qué futuro para la política de cohesión?». Información Comercial Española, n. 848 (2009): 115-124.

Wolff, Guntram. Beyond the Juncker and Schäuble visions of Euro-Area Governance. Policy Brief n. ${ }^{\circ}$ 6, Bruselas: Bruegel, noviembre 2017.

Yuill, Douglas, Encarna Murillo, María Jesús Delgado, Carlos Méndez. «Cohesion policy reform process: some implications for Spain and its regions», Presupuesto y Gasto Público, n. ${ }^{\circ} 55$ (2009):49-75.

Zubiri, Ignacio. «Las consecuencias presupuestarias de la ampliación de la Unión Europea». En La ampliación al Este de la Unión Europea. Editado por Ignacio Zubiri, 17-94. Madrid: Academia Europea de Ciencias y Artes, 2007.

\section{Sobre los autores}

Rafael Bonete Perales es Licenciado en Derecho, Licenciado en Economía, Licenciado en Sociología y Ciencias Políticas y ha estudiado un Máster en Estudios Europeos (Colegio de Europa. Brujas, Bélgica). En la actualidad es profesor Titular de Economía Aplicada en la Universidad de Salamanca y Titular de la cátedra Jean Monnet en Economía Europea. Sus publicaciones se han centrado en Economía de la UE y en Economía Pública, temas sobre los que ha publicado un número apreciable de artículos, capítulos de libros y libros editados por editoriales de prestigio como Alianza Editorial y Thomson-Civitas. Sus libros más importantes en temas europeos son: Condicionantes internos y externos de la PAC, 
ISBN: 84-491-0047-X,1994 e Introducción a la Unión Europea: un análisis desde la Economía (coautor con Rafael Muñoz de Bustillo), 978-84-2068277-8, cuarta edición, 2009.

Rafael Muñoz de Bustillo Llorente es Catedrático de Economía Aplicada en la Universidad de Salamanca. Entre sus estancias en el extranjero merece la pena destacar la de Senior Visiting Professor en la Universidad Northeastern, en Boston y la de European Scholar in Residence (spring semester), University of Massachusetts, Boston, en 1998. Sus investigaciones se han centrado en la Economía del Estado de Bienestar, mercado de trabajo, distribución de la renta y Economía de la UE, temas sobre los que ha publicado más de 170 artículos, capítulos de libros y libros editados por editoriales de prestigio como Alianza Editorial, Oxford University Press o Edgard Elgar. Entre sus aportaciones a la temática europea destaca Introducción a la Unión Europea: un análisis desde la Economía (con Rafael Bonete), que vio su cuarta edición en 2009. Dentro del análisis del mercado de trabajo merece la pena reseñar su Measuring more than Money. The Social Economics of Job Quality, publicado por Edgard Elgar en 2011. Ha sido investigador en múltiples proyectos europeos y de la OIT, el último de ellos sobre innovación y calidad de empleo dentro del programa Horizonte 2020 (Proyecto QuInnE). En 2017 fue galardonado con el Premio «María de Maeztu» de la Universidad de Salamanca a la excelencia científica.

\section{About the authors}

Rafael Bonete Perales holds a Bachelor's degree in Law, a Bachelor's degree in Economics, a Bachelor's degree in Sociology and Political Science and a Master in European Studies (College of Europe, Bruges, Belgium). Currently he is Associate Professor in the Department of Applied Economics at the University of Salamanca (Spain) and holder of a Jean Monnet Chair on European Economics. His main interests are European Economics and Public Economics, topics on he has extensively published articles, chapters of books and books edited by prestigious publishers, such as Alianza Editorial or Thomson-Civitas. His main books on European themes are: Condicionantes internos y externos de la PAC, ISBN: 84491-0047-X,1994 e Introducción a la Unión Europea: un análisis desde la Economía (co-author with Rafael Muñoz de Bustillo), 978-84-206-8277-8, 4th edición, 2009.

Rafael Muñoz de Bustillo Llorente is Professor of Applied Economics at the University of Salamanca. He has been Senior Visiting Profes- 
sor at Northeaster University in Boston in 2013 and European Scholar in Residence (spring semester) at the University of Massachusetts, Boston in 2018. His publications have dealt with many different issues from the economics of the Welfare State, the study of labour markets and income distribution to the economics of the European Union. He has more than 170 journal articles, chapters in collective books and books, including Introducción a la Unión Europea: un análisis desde la Economía (coauthored with Rafael Bonete), whose fourth edition was published in 2009, or Measuring more than Money. The Social Economics of Job Quality, published in 2011 by Edgard Elgar. He has participated as researcher in many EU and ILO projects, the current one, on innovation and job quality, financed by Horizonte2020 (Project QuInnE). In 2017 was awarded with the Prize María Maeztu of the University of Salamanca to excellence in research. 


\section{Derechos de autor}

Los derechos de autor (para la distribución, comunicación pública, reproducción e inclusión en bases de datos de indexación y repositorios institucionales) de esta publicación (Cuadernos Europeos de Deusto, CED) pertenecen a la editorial Universidad de Deusto. El acceso al contenido digital de cualquier número de Cuadernos Europeos de Deusto es gratuito inmediatamente después de su publicación. Los trabajos podrán leerse, descargarse, copiar y difundir en cualquier medio sin fines comerciales y según lo previsto por la ley; sin la previa autorización de la Editorial (Universidad de Deusto) o el autor. Así mismo, los trabajos editados en CED pueden ser publicados con posterioridad en otros medios o revistas, siempre que el autor indique con claridad y en la primera nota a pie de página que el trabajo se publicó por primera vez en $C E D$, con indicación del número, año, páginas y DOI (si procede). Cualquier otro uso de su contenido en cualquier medio o formato, ahora conocido o desarrollado en el futuro, requiere el permiso previo por escrito del titular de los derechos de autor.

\section{Copyright}

Copyright (for distribution, public communication, reproduction and inclusion in indexation databases and institutional repositories) of this publication (Cuadernos Europeos de Deusto, CED) belongs to the publisher University of Deusto. Access to the digital content of any Issue of Cuadernos Europeos de Deusto is free upon its publication. The content can be read, downloaded, copied, and distributed freely in any medium only for non-commercial purposes and in accordance with any applicable copyright legislation, without prior permission from the copyright holder (University of Deusto) or the author. Thus, the content of $C E D$ can be subsequently published in other media or journals, as long as the author clearly indicates in the first footnote that the work was published in $C E D$ for the first time, indicating the Issue number, year, pages, and DOI (if applicable). Any other use of its content in any medium or format, now known or developed in the future, requires prior written permission of the copyright holder. 